\title{
WestVirginiaUniversity
}

THE RESEARCH REPOSITORY @ WVU

Graduate Theses, Dissertations, and Problem Reports

2018

\section{Assessment of Surgical Guide Accuracy Utilizing a Digital Workflow}

Nicole Irene Andreini

Follow this and additional works at: https://researchrepository.wvu.edu/etd

\section{Recommended Citation}

Andreini, Nicole Irene, "Assessment of Surgical Guide Accuracy Utilizing a Digital Workflow" (2018). Graduate Theses, Dissertations, and Problem Reports. 5107.

https://researchrepository.wvu.edu/etd/5107

This Thesis is protected by copyright and/or related rights. It has been brought to you by the The Research Repository @ WVU with permission from the rights-holder(s). You are free to use this Thesis in any way that is permitted by the copyright and related rights legislation that applies to your use. For other uses you must obtain permission from the rights-holder(s) directly, unless additional rights are indicated by a Creative Commons license in the record and/ or on the work itself. This Thesis has been accepted for inclusion in WVU Graduate Theses, Dissertations, and Problem Reports collection by an authorized administrator of The Research Repository @ WVU. For more information, please contact researchrepository@mail.wvu.edu. 
Assessment of Surgical Guide Accuracy Utilizing a Digital Workflow

Nicole Irene Andreini, D.D.S.

\author{
Thesis submitted to the \\ School of Dentistry \\ At West Virginia University \\ In Partial fulfillment of the requirements \\ For the degree of \\ Master of Science \\ In \\ Prosthodontics
}

Matthew S. Bryington, D.M.D., M.S. (Chair)

Bryan D. Dye, D.D.S., M.S.

Bryan Weaver, D.D.S., M.D

Department of Restorative Dentistry

Morgantown, West Virginia

2018

Keywords: Surgical Guide Accuracy, Digital Planning Software, Intraoral Scanners (IOS), Digital Workflow for Implant Planning

Copyright 2018 Nicole Andreini 


\begin{abstract}
Assessment of Surgical Guide Accuracy Utilizing a Digital Workflow

Nicole Irene Andreini, D.D.S.
\end{abstract}

Objectives: To determine the accuracy of placing implants using a digital workflow. Variables were compared to see if there was an effect on implant position accuracy. These included bone level (BL) versus bone level tapered (BLT) implants, positioning of implants adjacent to tooth support versus further across an edentulous ridge, and impact of the surgical guide requiring adjustment to fully seat. Furthermore, two methods of post-operative assessment were compared to evaluate consistency of each approach.

Methods: A typical work up for digital implant planning was performed on a sectioned pig jaw. The DICOM CBCT file and IOS STL file were imported into coDiagnostiX to virtually plan implant placement (Dentalwings, Montreal, Canada). Two implants were planned for each of ten specimens, with one positioned adjacent to the Surgical guide tooth support (Implant A) and a second positioned more distally along the edentulous ridge (Implant B). The guide was designed and 3-D printed with $5 \mathrm{~mm}$ sleeves to allow full preparation of the osteotomy. The sites were prepared following Straumann guided surgery protocols, with the exception of utilizing irrigation. The implant was free-handed into position, until full depth of placement was achieved. The jaws were then post-operatively assessed using two methods: a post-operative CBCT scan and an intraoral scan of implant scan bodies. Each set of files were overlapped with the treatment evaluation tool of the coDiagnostiX software, to compare the planned to the resulting implant position.

Results: The average error was more pronounced at both the base and tip of implants in the mesial direction. An average 3D offset of $1.43 \mathrm{~mm}$ was observed at the coronal aspect, with a little higher average offset of $2.04 \mathrm{~mm}$ at the implant apex. Also, the 
average angular deviation was $5.17^{\circ}$. There was no significant difference found between the post-operative methods of assessment. Significant differences were shown between implant A and B when comparing the depth of placement. Differences were also found between BL and BLT implant types in angular deviation and in the mesial/distal direction at the platform of the implant. When the guide required adjustments, a significant difference in positioning was found in the buccal/lingual direction.

Conclusions: Post-operative assessment using a CBCT or IOS of scan bodies are comparable methods to evaluate planned versus placed implant positioning. Flexure of the surgical guide may have caused implants placed further from the tooth support to be positioned deeper than planned. BLT implants showed better angular accuracy than BL implants with a partially guided surgical approach. The accuracy of implant positioning utilizing a digital workflow in this study was found to be consistent with findings in the literature. 


\section{ACKNOWLEGEMENTS}

I would like to that the following for their guidance and contributions:

Dr. Matthew Bryington - I am so grateful for your instruction with this project. Thank you for providing me with the resources and training needed to complete my thesis.

Lyndsey Gray - Thank you for the tutorial on using the CBCT and autoclave.

Dr. Matthew Harper - Thank you for the idea to use an animal model for this area of research.

Dr. Gerry Hobbs - Thank you for your assistance with the statistical analysis.

Deanna Kritzer - Thank you for coordinating the collection of the pig specimens.

Austin Kritzer - Thank you for your help with sectioning and dissecting the pig jaws, to be suitable sizes for the parameters of this project.

I would also like to thank the following for their generous donations of materials for this project:

Davey Potter - Thank you for contributing the Straumann BL and BLT implants.

Rob Roche - Thank you for granting access to the "treatment evaluation" tool in coDiagnostiX, to allow post-operative comparison of implant positioning and providing surgical guide tokens for the generation of the guides. 


\section{TABLE OF CONTENTS}

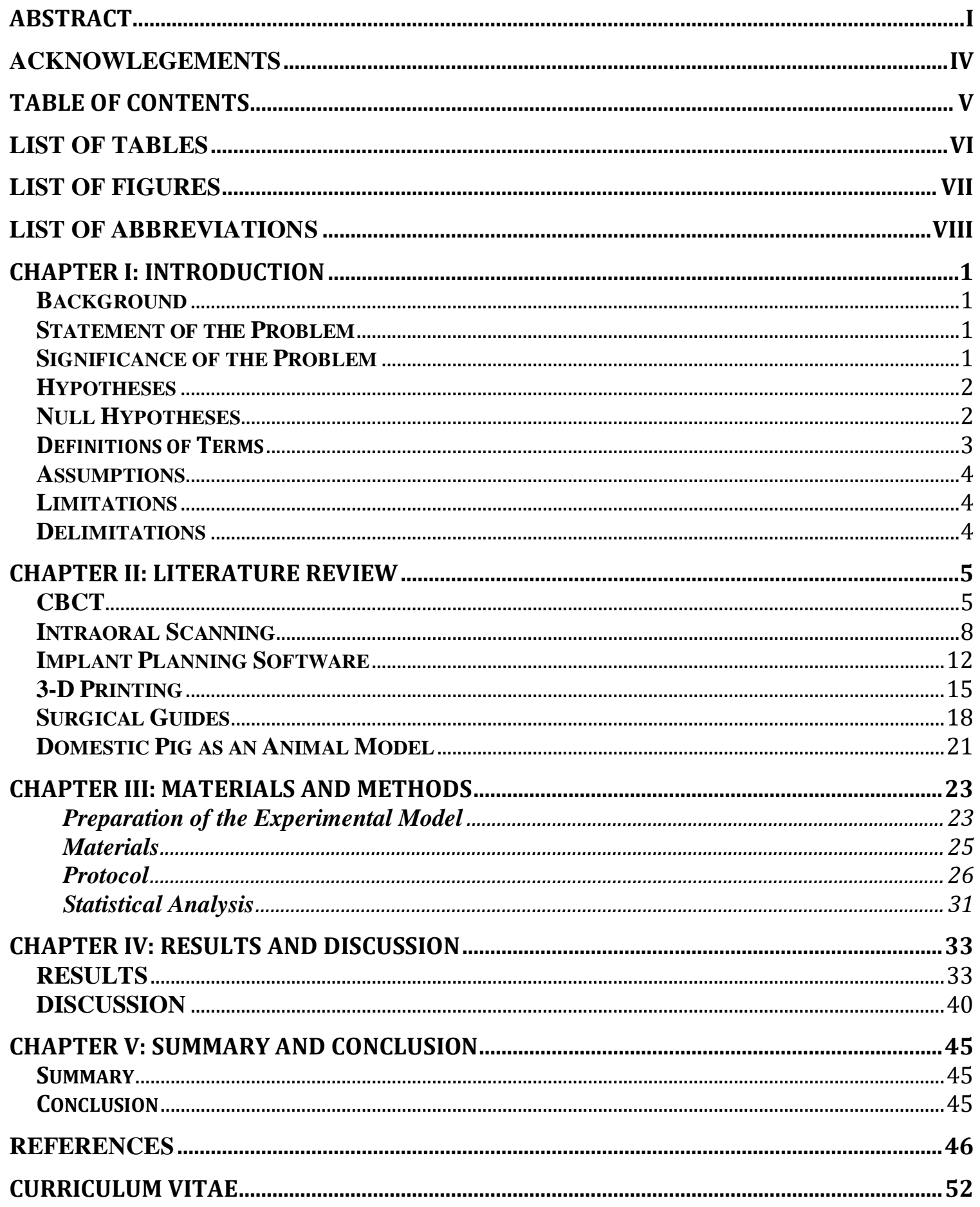




\section{LIST OF TABLES}

Table 1. Straumann $4.1 \mathrm{~mm}$ diameter implant descriptions according to guide number.

Table 2. Surgical guide and implant placement data.

Table 3. CBCT deviations of implant positioning.

Table 4. IOS deviations of implant positioning.

Table 5. Average discrepancies in each direction at the base and tip of implants, confidence intervals, Student's t-test values, and p-values.

Table 6. Student's t-test comparing implant position A and B.

Table 7. Student's t-test comparing implant type BL to BLT.

Table 8. Student's t-test assessing a difference in positioning if adjustment to the guide was required. 


\section{LIST OF FIGURES}

Figure 1 (A and B). Reduction in size of specimen: (A) Pig jaw after removal of tongue and dissection of surrounding muscles. (B) Sectioning of the posterior portion to provide a $10 \mathrm{~cm}$ length specimen.

Figure 2 A-C. Preparation of the pig jaws: (A) The jaws were sectioned at the midline. (B and C) The two specimens are shown, consisting of a right and left segment of one pig jaw.

Figure 3. Finalized digital design of surgical guide.

Figure 4 A-D. Surgical guide: (A) Lateral view. (B) Embossed implant types and lengths. (C) Occlusal view. (D) Embossed guide number.

Figure 5 A-C. CBCT treatment evaluation: (A) Alignment of pre-operative and postoperative segmentations. (B) Merging of the scans. (C) Manual positioning of the digital implant within the confines of the radiopaque implant from the CBCT.

Figure $6 \mathrm{~A}$ and B. IOS treatment evaluation: (A) Selecting the platform of the scan body for Implant A. (B) Treatment evaluation data.

Figure 7. Difference in distance between position A and B in the Base - Apical direction.

Figure 8. Difference in distance between position A and B in the Apex - Apical direction.

Figure 9. Difference between implant type BL and BLT in the Base - Distal direction.

Figure 10. Difference in angulation between implant types BL and BLT.

Figure 11. Difference between implant type BL and BLT in the Base - Vestibular direction.

Figure 12. Difference in distance if guide required adjustments. 


\section{LIST OF ABBREVIATIONS}

MSCT $=$ Multi-slice computed tomography

CBCT = Cone beam computed tomography

FOV $=$ Field of view

TFT $=$ Thin-film transistor

FDK method $=$ Feldkamp method

ALARA/ALADA = As low as reasonably/diagnostically acceptable

ICRP = International Commission on Radiological Protection

TLD $=$ Thermoluminescent dosimeter

MTF $=$ Modulation transfer function

IOS = Intraoral scanner

STL $=$ Standard Tesselation Language

POI $=$ Point of interest

AWS = Active Wavefront Sampling

CAI = Computer-aided implantology

DICOM = Digital Imaging and Communications in Medicine

CAD/CAM = Computer aided design/computer aided manufacturing

3DP $=3-\mathrm{D}$ printing

SLA = Stereolithography

$\mathbf{B L}=$ Bone level

BLT $=$ Bone level tapered

ANOVA = Analysis of varianc 


\section{Chapter I: Introduction}

\section{Background}

Development of CBCT imaging revolutionized the surgical approach to implant placement $^{1}$. It gave practitioners an in-office option for acquiring a three-dimensional radiograph for diagnostic assessment. It then became integrated with virtual planning software and CAD/CAM manufacturing of surgical guides ${ }^{1}$. This provided an improved degree of accuracy, compared to a free-handed approach ${ }^{2}$. Furthermore, it helped minimize surgical complications due to proximity of various anatomical structures $3 ; 4$. The guided approach also allowed for flapless surgeries, which was key for cases in the esthetic zone with limited mesio-distal/bucco-lingual space and thin gingival biotypes $5 ; 6$.

Intraoral scanners have now become a more prominent tool for implant dentistry as well. They are commonly used to capture a digital impression of a tooth or an implant scan body to send to the lab for digital designing and fabrication ${ }^{7}$. However, their use as a tool for guided surgery has limited support in the literature ${ }^{8}$. Also, few variables within a specific guide type have been further assessed.

\section{Statement of the Problem}

To what degree of accuracy do stereolithographic tooth-supported surgical guides, fabricated from a digital workflow, relate planned implant positioning to the clinical situation? What impact does implant positioning, implant type, and adjustments to the surgical guide have on implant placement accuracy?

\section{Significance of the Problem}

Guided implant placement improves positioning accuracy and minimizes surgical complications for a broad range of $\operatorname{cases}^{3-6 ; 9}$. However, it is challenging to determine clinical accuracy and precision of implants placed using stereolithographic guides, due to 
the various systems and workflows available in the market ${ }^{6}$. Currently, little knowledge is presented on the source and degree of inaccuracies of a fully digital workflow in the literature ${ }^{10}$. Each step incorporates minimal errors that can accumulate to result in larger total error. Understanding the intrinsic errors of the process and how to minimize human errors, can help clinicians to maximize position accuracy and properly plan to account for its limitations. This study focused on the accuracy of implant placement utilizing a digital workflow for surgical guide fabrication. It further assessed different variables of tooth-supported surgical guides and their effects on implant placement accuracy.

\section{Hypotheses}

The two methods of post-operative assessment will show a significant difference in results. The position of the implant (in relation to the tooth support), the type of implant used, and adjustment of the surgical guide will affect the accuracy of implant placement.

\section{Null Hypotheses}

There will not be a difference in the results of the two methods of post-operative assessment. There will be no difference in implant placement accuracy relative to implant position, implant type, or adjustments to the surgical guide. 


\section{Definitions of Terms}

$\mathbf{C A D} / \mathbf{C A M}=($ Computer Aided Design/Computer Aided Manufacturing $)$ a technology that allows clinicians to digitally create dental products and relates design information to allow fabrication of the virtual plan ${ }^{11}$

Scan body $=$ virtual impression copings that are digitally scanned to relate implant position to a third party, typically for fabrication of a restoration

3-D volume rendering $=$ digital translation of a three-dimensional scan into twodimensional images, for viewing on a computer

Segmentation $=$ the manual process (performed using implant planning software) of adjusting the gray values, and eliminating scatter or other artifacts present in a scan for optimal visualization ${ }^{9}$

Tolerance $=$ the differences in diameter of the sleeve-related components of the guided surgery system ${ }^{12}$

Intrinsic error $=$ error attributed to the mechanical design features of the guide ${ }^{12}$

Fully guided $=$ surgical approach where a $\mathrm{CAD} / \mathrm{CAM}$ surgical guide is used to prepare the implant site and the implant is placed through the template as well ${ }^{13}$

Partially guided $=$ surgical approach where a $\mathrm{CAD} / \mathrm{CAM}$ surgical guide is used to prepare the implant site, but the implant itself is placed in a "free-handed" way ${ }^{13}$ 


\section{Assumptions}

1. It is assumed that the pig jaws were appropriately positioned in the CBCT and digitally oriented in the coDiagnostiX software.

2. It is assumed that both BL/BLT implants and implant transfer copings were homogenous to their respective implant type in size and structure.

3. It is assumed that the scan bodies were uniformly seated during post-operative IOS scanning.

\section{Limitations}

1. Sample size of ten specimens with a total of twenty implants for statistical analysis.

2. Burs were not replaced after ten surgeries (as recommended by Straumann protocols), due to cost.

\section{Delimitations}

1. Limited to tooth-supported surgical guides.

2. Limited to findings involving one implant system.

3. Limited to an animal model. 


\section{Chapter II: Literature Review}

\section{CBCT}

The first diagnostic requirement for a digital workflow for implant planning, is acquiring the appropriate radiograph. Intraoral peri-apical radiographs have a high spatial resolution, which makes them useful for diagnosing caries and tooth-related pathologies ${ }^{14 ; 15}$. However, these images are small and have anatomic overlap, which limits their use for implant planning purposes ${ }^{14 ;}$. Panoramic radiographs are useful for assessing bone height, nerve location, and position of the sinuses, as a preliminary assessment to evaluate potential implant sites ${ }^{4 ; 14}$. However, the substantial distortion (approximately 25\%), anatomic overlap, limited resolution, and the two-dimensional nature of the image, make panoramic radiographs a poor option for implant planning ${ }^{14-16}$.

The options for three-dimensional dental imaging include conventional computed tomography (CT), multi-slice computed tomography (MSCT), and cone beam computed tomography (CBCT). Conventional CTs were invented by Hounsfield in $1972^{14}$. They are digital images created from multiple exposures of a fan-shaped x-ray beam at different angles through a tissue slice. Conventional CTs are shown to yield image accuracy similar to CBCTs and MSCTs, but at a greater cost, with increased acquisition time, greater radiation doses, and increased technique sensitivity ${ }^{17-20}$. Therefore, they've been deemed "unsuitable for clinical practice"17. Instead of using the one-dimensional detector arcs of conventional CTs, multi-slice CTs use multiple detector rows (up to 320) in a two-dimensional array. This results in higher image resolution with shorter scanning times, but requires multiple rotations ${ }^{14}$. This leaves cone beam CTs, which use a coneshaped source of ionizing radiation. They were specifically developed for the maxillofacial region as an alternative to conventional CTs, to more efficiently capture the data set with a less expensive detector ${ }^{17}$. CBCTs use smaller voxels than conventional CTs and allow adjustment of the field of view (FOV), which results in higher spatial

resolution ${ }^{17 ; 21}$. They scan the patient (similar to a panoramic radiograph) in one rotation with a low radiation dose, but are shown to have increased noise and lower contrast resolution ${ }^{14}$. 
Cone-beam CTs are currently the predominant means of obtaining threedimensional imaging for implant planning ${ }^{18 ; 19}$. A cone-shaped beam emanating from the $\mathrm{x}$-ray source projects onto a detector and rotates around the center of the region of interest, capturing multiple sequential planar projection images ${ }^{19}$. The flat-panel detectors consist of a large-area pixel arrangement of hydrogenated amorphous silicon thin-film transistors (referred to as TFTs) and a photo diode array. The x-rays are detected indirectly by a scintillator, which translates the information into visible light that is registered in the photo diode array. The TFTs are the carriers of that signal information. Typically, the rays are pulsed to correspond with detector sampling, which minimizes exposure time and therefore radiation dose. The multiple two-dimensional images captured, resemble that of a lateral cephalometric radiograph and are referred to as raw, basis, and/or frame images ${ }^{18 ; 19}$. Together, these images comprise the total projection data. The data is converted into a viewable format by a process termed reconstruction, using a mathematical algorithm called the FDK (or Feldkamp) method. More projection data gives greater spatial and contrast resolution, and increases the signal-to-noise ratio, resulting in "smoother" images and reduction of metallic artifacts ${ }^{16}$. However, this means a higher exposure dose which needs to be limited in accordance with ALARA/ALADA (as low as reasonably/diagnostically acceptable) ${ }^{19}$.

CBCT radiation doses vary according to the unit used, FOV, milliamp setting, voxel size, scan time and type (continuous vs pulsed), sensor sensitivity, and the number of captured images $22 ; 23$. The International Commission on Radiological Protection (ICRP) conducts dose studies using human cadaver skulls with calibrated thermoluminescent dosimeters (TLDs) to assess the absorption of surrounding radiosensitive anatomy ${ }^{23}$. They then use this information to obtain the effective dose in microsieverts as a resource for clinicians. Also, the ADA council on Scientific Affairs recommends that dose optimization is observed when choosing settings for CBCT scans $^{22}$. Clinicians should aim to limit the radiation dose by selecting the appropriate FOV, shortening scan times, and reducing the milliamp setting ${ }^{22 ; 23}$. Care should be taken to minimize the risk to the patient without compromising the diagnostic value of the scan. 
To use a CBCT as a diagnostic tool for implant planning, it is important to understand the accuracy of the resulting images. Widmann et al used implant positioning as a way to evaluate the KaVo 3D eXam CBCT unit's accuracy, and found a mean error of $0.36 \mathrm{~mm}^{24}$. Another study compared actual measurements of a cadaver skull taken using a caliper, to digital measurements taken from four different CBCTs to determine device specific errors. There was a significant difference between the actual and digital measurements, with the digital showing a slight over-estimation ${ }^{25}$. One of the units assessed in this article is the same machine that was used in the present study, the Carestream CS 9300 (Carestream Dental LLC, Atlanta, Georgia). The overall mean deviation was $0.229 \mathrm{~mm}$, when assessing the Carestream CS 9300 set to a FOV of 8 X 8 $\mathrm{cm}$, tube voltage of $90 \mathrm{kV}$, and tube current intensity of $3.2 \mathrm{mÅs} 25$.

A CBCT's detail accuracy can be reduced approximately $0.100-0.150 \mathrm{~mm}$ by the modulation transfer function (MTF), the volumes voxel size, and the algorithm of gray values between the voxels ${ }^{5}$. Upon transferring the data set to a software viewer, differences of $0.5 \mathrm{~mm}$ between different devices have been reported ${ }^{5}$. The overall performance depends on technical specifications and reconstruction algorithms, with the reconstruction algorithms showing the highest impact on accuracy ${ }^{5}$. However, there are other potential sources of error to contribute to CBCT image discrepancies.

Artifacts can occur, such as beam hardening, due to metal fillings, crowns, and implants $^{14}$. The high density of the metallic materials reduces the image quality, creating white and black rays surrounding the site. Also, partial volume averaging may result if the voxel resolution is greater than the spatial/contrast resolution of the FOV ${ }^{16}$. An example of this is the shadowing that surrounds implants, due to inconsistencies between views. These partial volume averaging artifacts can be minimized by selecting smaller voxels. Peripheral areas of the scan volume can be distorted as well, due to the divergence of the beam as it rotates. That means there is less information detected for the peripheral structures, while more information is focused on the central objects ${ }^{16}$. The patient may even move while the device is capturing the images. This can be minimized by reducing the scan time and with the use of a head restraint ${ }^{16}$. Furthermore, alterations in patient positioning can lead to inaccurate measurements that can compromise implant 
planning ${ }^{26}$. Visconti et al assessed measurement variations comparing a standard position to deviations of $10^{\circ}$ and $20^{\circ}$ in superior, inferior, and lateral directions. The study found a significant difference in measurements for nearly every variation in position. The $20^{\circ}$ superior position had the greatest discrepancies, with the majority of errors over-estimating the true values. Lateral displacement distorted the image on the contralateral side, affecting the posterior the most. They concluded that mal-positioning during CBCT image acquisition results in alterations of bone height and width measurements that can result in errors in implant planning ${ }^{26}$.

\section{Intraoral Scanning}

The next source of information needed for surgical guide fabrication, is an impression of the patient's appropriate arch. This can be achieved by making a conventional impression or capturing an intraoral scan. There are several studies to show that patients prefer the digital impression approach ${ }^{7 ; 8 ; 27}$. All of them indicated that intraoral scanning resulted in reduced anxiety and nausea response compared to a conventional impression (regardless of material type $)^{7 ; 8 ; 27}$. It also allowed for better communication with patients, since they could view the image of their dentition on the screen with the clinician ${ }^{8}$. This has been shown to make the patient feel more involved with their treatment and even have a positive impact on patient compliance (indicated by improved oral hygiene) $)^{8}$.

Another advantage of the digital approach is overall efficiency. There is some debate over this issue according to a systematic review conducted by Gallardo et $\mathrm{al}^{7}$. Of the five included articles, two reported less time for the conventional approach. However, it is worth noting that the conventional approach used in one of these studies was a "triple tray" technique, while the second mentioned the operator had difficulty capturing the interproximal portions of scan bodies for a digital implant impression ${ }^{7}$. The rest of the literature agrees that obtaining the scan itself is not necessarily faster than a conventional approach, but the fact that a scan does not require the additional steps of pouring a cast and trimming a model increases its efficiency ${ }^{8}$. Furthermore, if there is an 
inaccuracy in the intraoral scan, it is easily corrected by deleting only the error and rescanning the site ${ }^{7 ; 8}$. A conventional impression would need to be remade.

The digital approach can also simplify the procedure for the clinician. In the case of a severe undercut or diverging positions of multiple implants, an intraoral scan would be much easier to obtain versus attempting to remove a conventional impression ${ }^{8}$. Research has also shown that clinicians with an affinity for technology will easily adapt to using an $\operatorname{IOS}^{8 ; 27}$. However, this can also be dependent on the technology used by various intraoral scanners ${ }^{8}$.

Optical impressions were created by Dr. Francois Duret in $1971^{28}$. About a decade later, Professor Mormann from Switzerland designed and patented the first handheld intraoral scanner (IOS) that he called the "Chairside Economical Restoration of Esthetic Ceramics" or the CEREC ${ }^{28}$. IOS units, like the CEREC, typically consist of a handheld camera connected to a mobile cart, containing a computer equipped with software to translate the acquired data into an image ${ }^{27}$. The most widely used digital format for this information is the Standard Tesselation Language (STL) ${ }^{27}$. It contains a succession of triangulated surfaces, where each triangle contains the data of its three points and a normal surface ${ }^{27}$.

There are different types of imaging technology, but all of them have certain features in common. All cameras require light projection to record either individual images or a video, that is then compiled by the software using points of interest (POI) ${ }^{27}$. The POIs are defined by three coordinates: two coordinates ( $\mathrm{x}$ and $\mathrm{y}$ ) that can be assessed on the image, and a third coordinate (z) that requires an algorithm to determine ${ }^{27}$. This third coordinate is calculated using different "distance to object technologies" depending on the IOS system. Most of these systems use an active light projection technique, meaning a white, red, or blue structured light is projected from the camera onto the object. This is advantageous because the camera is less reliant on texture and color of the object for reconstruction. The alternative, passive light projection, uses only ambient light and requires a certain level of texture of the object ${ }^{27}$. 
There are four main "distance to object technologies" (or z-coordinate determinants $)^{27}$. There is triangulation, where a POI is viewed from different angles and this data (distance from POI, angles of the two points of view, etc.) is used in a formula to calculate the positioning. Another method is confocal imaging, which is based on acquisition of focused and defocused images from different depths. The software can detect the sharpness area of the image to gauge the distance to the object. It is then reconstructed using successive images taken at different focuses, aperture values, and from various angles around the object. A third distance to object technology is Active Wavefront Sampling (AWS). This technique requires an off-axis aperture module that moves in a circular path, which essentially rotates the aperture to skew a POI captured by the camera. This results in a pattern produced by each point that is used to calculate distance and depth. The last, and only technique to use passive light projection, is stereophotogrammetry which estimates all three coordinates $(x, y$, and $z)$ through an algorithmic analysis of images.

After the three coordinates of multiple POIs are defined, a reconstruction is required to merge the images of the intraoral scan. Differences between the images can be calculated by an accelerometer in the camera or a similarity calculation to assess the image point of view ${ }^{27}$. A transformation matrix is formed based on similarities between the images, such as rotation or isotropic scaling of POIs.

The quality of the images can sometimes be compromised, due to the reflective surfaces of the oral environment, making POI matching difficult. The clinician can attempt to change the orientation of the camera, to increase the diffuse light and illuminate these surfaces. Some systems help counteract this problem by having a polarizing filter in the camera, while others use a 20 to $40 \mu \mathrm{m}$ powder coating on the object to reduce the reflectivity. There is a concern that the thickness of the powder could reduce accuracy. However, the IOS software takes an average thickness into account in its calculations ${ }^{27}$.

This leads to a discussion of just how accurate different IOS systems are and how they compete with the accuracy of conventional impressions. Ender et al compared the 
precision of several conventional impression materials and multiple digital systems for generating complete arch dental impressions in vivo. Systems utilizing active triangulation were found to have the same high precision of conventional impressions taken with polyether or vinylsiloxanether. Irreversible hydrocolloid was shown to have the lowest precision. Some trends were identified, such as regions of the arch with lower precision. Video-based digital impression systems progressively deformed as the scan moved further posteriorly. Still-image IOS systems showed a similar trend but with regionalized deviations as well. Conventional impression techniques showed local deviations, but there were no regions showing a noticeable increase in magnitude. The authors hypothesized that the trends could be attributed to the little geometric information available in the anterior region. This could contribute to an error propagation that appears more pronounced toward the distal ends of the $\operatorname{arch}^{29}$.

Another study by Nedelcu et al assessed both the accuracy and precision of $3 \mathrm{M}$ True (stereophotogrammetry/video-based), Trios 3 (triangulation/video-based), and Omnicam (triangulation/video-based) IOS systems versus a conventional polyether impression $^{30}$. This in vivo study is a rarity for this subject in the literature, due to the difficulty in determining an accurate reference model. An industrial grade scanner (ATOS Core 80) was used to acquire the reference scan as the basis for comparison of accuracy. Also, the final model (poured using Type IV dental stone) produced from the conventional impression was scanned with the D1000 laboratory scanner. This allowed the conventional impression to be formatted to an STL file in order to provide similar formatting of all the impressions. These images were overlapped using a powerful reverse-engineering software to generate colorimetric maps to show ranges of differences in specific regions of the $\operatorname{arch}^{8 ; 30}$. The average deviations of both the ATOS Core 80 and D1000 lab scanner were confirmed to be negligible $(<1 \mu \mathrm{m})$. There was no significant difference in precision among the various IOS scanners. When comparing accuracy, $3 \mathrm{M}$ True and Trios 3 were found to be significantly more accurate than the Omnicam IOS, while the conventional polyether impression overlapped with both groups (displayed no significant difference). The Omnicam regions of inaccuracy included positive deviations in the premolar areas with negative deviations in the frontal area. 
A study by Ji-man Park assessed both accuracy/trueness and precision of various IOS systems using a laboratory dental reference. The IOS systems used either still image or video acquisition methods, and either active triangulation (requiring the use of powder) or confocal microscopic technology. Those IOS systems utilizing active triangulation with powder were shown to have significantly better trueness than IOS systems utilizing confocal microscopic technology. There were significant differences among precision of the scanners, but it was not attributed to the scanner technology of the IOS system. There was also no difference in trueness or precision between still-image versus video-image acquisition of IOS systems. The overall average deviations in trueness were $70.1 \mu \mathrm{m}$ and deviations in precision were $58.9 \mu \mathrm{m}^{31}$.

Therefore, the literature does not support one specific scanning technique, IOS scanner, or technology as more accurate due to heterogeneity of procedures and insufficient in vivo studies ${ }^{27}$. The trueness and precision depend on aspects such as the operator, system used, duration of scan, and the oral environment ${ }^{27}$. There is a consensus when it comes to fabrication of restorations, that IOS units demonstrate similar or higher accuracy than conventional impressions when it comes to single units and quadrant dentistry ${ }^{27 ; 28}$. However, there are more pronounced deviations in precision and trueness when it comes to full arch scans $27 ; 29 ; 30$. Based on a narrative review conducted by Mangano et al in 2017, it is recommended that care is taken when using IOS for guided surgery since it is a technology that is still "only in its infancy".

\section{Implant Planning Software}

Beginning in 1997, computer-aided implantology (CAI) software programs became available for interpretation of CBCT Digital Imaging and Communications in Medicine (DICOM) files ${ }^{4 ; 5}$. Once loaded into the software, the radiographic scan can be viewed in cross-sectional images to assess the surrounding anatomy and digitally plan the positioning of implants $\mathrm{s}^{3-5}$. The planned position can then be related to the clinical situation by virtually designing and 3-D printing a stereolithographic surgical guide ${ }^{6 ;} 9$. This technique is especially useful for challenging cases with limited horizontal/vertical 
bone height, close proximity to adjacent teeth, and close proximity to various anatomical structures (maxillary sinus, inferior alveolar nerve, etc.) ${ }^{3 ; 5 ; 6 ; 9 ; 32}$. It also gives the option of performing a flapless surgery, which is critical for difficult esthetic cases with thin gingival biotypes ${ }^{5}$. The ultimate goal of guided implant placement is to facilitate proper implant positioning in the bone to maximize both surgical and esthetic success ${ }^{6}$.

To begin planning, the CBCT DICOM file must be imported into the software program $6 ; 9$. Then the scan should be oriented in each plane: sagittal, axial, and coronal. The right or left Curve of Spee is oriented in the sagittal view to be level with the horizontal axis. Proper positioning in this view is most important ${ }^{6}$. If the arch is tilted incorrectly, it results in oblique slices in the cross-sectional view ${ }^{6}$. This distorts the image, creating an exaggerated view for evaluating vertical measurements ${ }^{6}$. Next, the axial orientation should be adjusted to center the patient's midline, focusing on symmetry of the arch form. It is useful to align the anterior and posterior nasal spines of the maxilla for more accurate positioning ${ }^{6}$. Lastly, the arch should be positioned in the coronal view by leveling the occlusal plane, visualizing the Curve of Wilson to achieve horizontal symmetry of the right and left sides ${ }^{6}$. The final step of orientation is creating the crosssectional views. The main principle when using this feature, is to create a curve where the cross-sectional imaging will be perpendicular to the planned implant sites while still following the level of the occlusal plane. Poor positioning of the curve can create oblique cuts in the bucco-lingual dimension that over-estimate horizontal bone volume ${ }^{6}$. However, if all the orientation steps are followed appropriately, the measurements surrounding planned implants will be accurate in both the buccal-lingual and inferiorsuperior direction ${ }^{6}$.

Often times, the 3-D volume rendering requires segmentation to remove scatter created from metallic restorations ${ }^{6 ; 9}$. The initial step is to adjust the bone pixel or gray value thresholds to isolate soft tissue from the bone ${ }^{5 ; 6}$. Removing the artifacts is a more tedious process, requiring the creation of layers or use of removal tools to outline the tooth forms ${ }^{6}$. It is important to recognize that these images do not illustrate the internal anatomy ${ }^{6}$. However, it is very useful for aligning the CBCT DICOM file with an intraoral scan STL file. 
The segmented CBCT volume rendering is then merged with an imported STL file of the patient's dentition ${ }^{4}$. This step also requires great attention to detail, since the STL file creates the virtual model used for the internal design of the surgical guide ${ }^{9}$. The file is super-imposed onto the scan by selecting corresponding reference points on each image until an accurate alignment can be performed by the software ${ }^{4}$. If the scatter of the 3-D rendering of the CBCT is not effectively removed during segmentation, the surface alignment could have a potential $0.45 \mathrm{~mm}$ discrepancy ${ }^{5}$.

A study by Flugge et al assessed the effect that imaging artifacts and clinician experience had on registration accuracy ${ }^{9}$. The implant planning software used was the same as that of the current study, coDiagnostiX (Dentalwings, Montreal, Canada) ${ }^{9}$. Two experienced and two inexperienced dentists worked on four different CBCT scans with an increasing number of restorations ${ }^{9}$. They first created a default segmentation of each scan provided by the software, with preset gray values ${ }^{9}$. Then, they created a manual registration with adjusted bone pixel thresholds and corrections using the various segmentation tools ${ }^{9}$. These different segmentations were aligned by the dentist with the CBCT's corresponding IOS STL file. The accuracy of the registration was compared by measuring the gap between the files at defined landmarks ${ }^{9}$. The results showed an overall deviation of $0.54 \mathrm{~mm}$, with the default alignment $(0.69 \mathrm{~mm})$ being significantly less accurate than the manually segmented registration $(0.40 \mathrm{~mm})$. Researchers also found that an increase in the number of restorations showed an increase in deviations of the models ${ }^{9}$. However, experience did not have a significant influence on the accuracy of registration 9 .

After the imported STL file is aligned with the CBCT, the clinician can trace the inferior alveolar nerve of the mandible ${ }^{6}$. This can be challenging for patients that are osteopenic or if there are motion artifacts present, since the canal is not usually well defined $^{6}$. It is also important to view the outline in all three planes, since the positioning can deviate at the lingual and mental foramina ${ }^{6}$. Another helpful feature, is the ability to adjust the width of the canal for a custom tracing of that individual patient's anatomy ${ }^{6}$. This identification of the nerve allows the software to alert the clinician when the planned implant position is within $2 \mathrm{~mm}$ of the outlined canal ${ }^{6}$. 
At this point, the virtual implant planning can begin. The software has a library of multiple implant systems, types, widths, and diameters from which to choose. The specific site can be assessed to visualize the location of the nasal floor, maxillary sinus, nasopalatine canal/foramen, inferior alveolar canal, extent of an anterior loop (present in $75-88 \%$ of patients), and/or the median lingual vascular canal (found at the lingual midline $)^{6}$. Most programs also provide a rendering of the bone volume ${ }^{4}$. This can be used to gauge the relative bone density, which is based on the software's interpretation of the gray values and calibration of the $\mathrm{CBCT}^{4}$. This information can be helpful for selection of implants that cater to lower density bone, and for prediction of primary stability on the day of the surgery ${ }^{4}$. Another helpful feature is the ability to digitally insert final abutments to optimally position restorative margins or screw access channels, which is especially beneficial for planning fixed detachable prostheses ${ }^{4}$. All of these tools, combined with optimal visualization, provide a precise virtual plan for implant placement. Once a plan is finalized, the software uses vectors of the implant positions to align guidance-sleeves for the design of the surgical guide ${ }^{5}$.

\section{3-D Printing}

The ability to print surgical guides is a more recent advancement in the technology of digital dentistry ${ }^{33 ; 34}$. CAD-CAM guides have been known to be the most accurate, but had been the least used due to cost, preparatory work, difficulty navigating planning software, and lab turnover times ${ }^{33}$. Following the expiration of certain patents, new 3-D printing methods developed and the technology is currently more efficient and affordable ${ }^{2 ; 34}$.

There are five types of 3-D printing that are relevant in dentistry: extrusion printing, inkjet printing, laser melting, 3-D printing (3DP), and stereolithography (SLA) printing ${ }^{34 ; 35}$. Extrusion printing involves the controlled dispensing of material through a nozzle, while a three-axis stage moves as programmed by a computer ${ }^{34}$. Inkjet printing also uses a three-axis stage, but the material is dispensed as micrometer sized drops ${ }^{34}$. Laser melting is another additive technique utilizing a high temperature laser light to 
manipulate a powder on a printing platform. The design is printed layer by layer as the platform moves either up or down ${ }^{34}$. 3DP is the only subtractive technique discussed, where material is removed by water jetting and repositioning of the object ${ }^{35}$. Stereolithography printing is the most common method in the dental field ${ }^{33}$. A photosensitive resin bath is manipulated with the use of a laser at a specific wavelength. The design platform starts in the resin bath and is directed away as programmed, while the planned design is sequentially built ${ }^{33-35}$. There are now desktop models available for SLA printing, which allow in-office fabrication of dental surgical guides ${ }^{36}$.

These printers have been shown to be competitive with laboratory and manufacturer fabricated guides $2 ; 11 ; 37$. A study using a digital workflow, analyzed 84 surgical guides and found that $93 \%$ of the intaglio of the guides were within $100 \mu \mathrm{m}$ of accuracy ${ }^{2}$. A case study aimed to determine the final error attributed to the printed guide, by superimposing an STL of the printed guide to the planned file. The maximum deviation was consistent with the previous analysis, equaling $0.1 \mathrm{~mm}^{2}$. Studies show that the resulting implant positions (placed with an SLA printed guide) have an average deviation of $0.9-1.0 \mathrm{~mm}$ and an average $5^{\circ}$ angulation error. This level of accuracy has far exceeded free-hand techniques, which show a deviation of $2.0-2.5 \mathrm{~mm}$ on average ${ }^{2}$; $20 ; 38 ; 39$. Even when an experienced clinician is performing the implant surgery, SLA guided results are significantly more accurate and predictable ${ }^{2 ; 6 ; 37 ; 40}$. Laboratory castfabricated surgical guides also show greater discrepancies than SLA manufactured guides, with an average $1.5 \mathrm{~mm}$ positioning error and $8^{\circ}$ angulation error ${ }^{2 ; 1}$.

The orientation of printing has been shown to affect accuracy using SLA methods ${ }^{34}$. Tahayeri et al printed samples with a specific dimension at $0^{\circ}, 15^{\circ}, 45^{\circ}$, and $90^{\circ}$ orientations using a FormLabs $1+$ printer. The overall percent error ranged from 0.12 to $2.4 \%{ }^{34}$. Objects printed at a $90^{\circ}$ orientation had a significantly lower percent error than those printed at a $45^{\circ}$ orientation ${ }^{34}$. When assessing thickness accuracy of materials, $0^{\circ}$ orientation was significantly more accurate than $90^{\circ}$ orientation. For the purposes of surgical guide fabrication, the thickness accuracy is not a priority. However, the internal fit is important to effectively communicate the planned implant position to the clinical 
setting. While printing a surgical guide at $90^{\circ}$ would require a longer print time, it may be beneficial when trying to minimize error.

Another potential source of error may be the metal sleeve of the drill guide $6 ; 10$. One study set out to determine the percentage of error caused by the tolerance between the metal sleeve of the guide and the drill key, combined with the tolerance between the drill key and the drills themselves ${ }^{12}$. The systems used were the External Hex Safe guide and the Safe System Surgical Kit. A theoretical "intrinsic error" was calculated using the known amount of tolerance between each of $0.20 \mathrm{~mm}$, and the height of the sleeve or drill key ${ }^{12}$. The resulting combined total angular deviation due to the guide's intrinsic error was $5.15^{\circ}$. When factoring this value into the final mean angular implant deviation of the study, the theoretical error accounted for $62.6 \%$ of the discrepancy ${ }^{12}$.

Schneider et al also tested the deviations that occurred due to the metal sleeve in SLA guides ${ }^{10}$. This study compared a guide with the manufacturer-issued metal sleeve, to a guide without a sleeve. This guide was printed to account for the size of the drill keys, at a reduced sleeve diameter ${ }^{10}$. The tolerances of each were challenged by applying $4.2 \mathrm{Ncm}^{2}$ of lateral force to maximize movement of the drill tip. It was found that lateral movement was reduced by $43 \%$ when the metal sleeve was eliminated ${ }^{10}$. Another study attempted to minimize the intrinsic error by using a direct drill guiding implant system ${ }^{41}$. The drills in this article were designed with modified shanks, to eliminate the need for drill keys. The sleeves of the guide were only $0.05 \mathrm{~mm}$ smaller in diameter than the modified shanks (compared to the typical $0.15-0.2 \mathrm{~mm})^{12 ; 41}$. The results showed that this approach resulted in two times better accuracy than other more conventional systems ${ }^{41}$. Therefore, the tolerance of the components guiding the drill have a significant impact on the accuracy of guided surgery ${ }^{12 ; 41}$.

Sleeve length and implant length can also play a role in positioning accuracy $6 ; 10$; 12; 42 . The use of longer guide sleeves and shorter implants has been shown to significantly reduce the amount of angular deviation $6 ; 10 ; 12 ; 42$. The additional length of the sleeve likely reduces the tolerance and limits the angular movement of the drill. Also, using a smaller implant decreases the depth of reduction, minimizing the extent of apical 
error. This means that the distance from the guide sleeve to the planned implant platform can also effect accuracy. It has been found that a greater gap is associated with a greater overall discrepancy between the planned and placed implant position ${ }^{12}$.

Some areas of concern regarding CAD-CAM surgical guides, include heat generation. The guides can restrict irrigation; however, the resulting temperature does not come close to inducing bone necrosis ${ }^{37}$. Routine sterilization procedures can also create problems. The high temperatures used in autoclaves have been shown to distort the SLA guide material ${ }^{43}$. Also, problems can arise when a provider relies too heavily on a surgical guide. There can be issues with fit or digital errors that compiled to create a poor translation of the virtual plan ${ }^{37}$. If the surgeon does not continue to verify the virtual plan with clinical situation, greater errors can occur.

\section{Surgical Guides}

There is much controversy in the literature surrounding which type of surgical guide is most accurate: bone-supported, mucosa-supported, or tooth-supported.

Information regarding accuracy of other variables for guided surgery are somewhat more consistent, such as surgical approach (flap or flap-less), stabilization techniques (use or disuse of fixation screws), location (maxillary or mandibular arch), and performing partially or fully guided implant placement $6 ; 12 ; 13 ; 20 ; 44-46$. For the purposes of this study, a tooth-supported surgical guide was used. This included a flap-less approach in a pig mandible without the use of fixation screws, and applying a partially guided protocol.

When assessing surgical guide type, it is difficult to reach a definitive conclusion regarding accuracy. Turbush et al compared the accuracy of implant placement using the three guide types in vitro with acrylic resin mandibles ${ }^{47}$. There were significant differences found in deviations at the implant platform and apex. While both the boneand tooth-supported guides were significantly more accurate than the mucosa-supported guide at the implant platform, only the tooth-supported guide was more accurate at the apex ${ }^{47}$. This led the authors to conclude that the tooth-supported was the most accurate surgical guide. This conclusion was reinforced with an in vivo study conducted by Ozan 
et al, again assessing all three surgical guide types ${ }^{48}$. They found that tooth-supported surgical guides resulted in significantly more accurate positioning at the implant apex than both bone- and mucosa-supported guides. In this case, a significantly greater angular deviation was found with the bone-supported guide than the tooth-supported guide $^{48}$. Both studies concluded that tooth-supported surgical guides were superior in accuracy; however, the in vitro study found the bone-supported guide to be slightly more accurate than the mucosa-supported guide, while the in vivo study found the mucosasupported guide to yield marginally better results than the bone-supported guide. Interestingly, an in vivo study by Cassetta $e t$ al found that mucosa-supported guides were superior in accuracy, for coronal and apical deviations, to both tooth- and bone-supported surgical guides ${ }^{45}$. They also concluded that mucosa-supported guides yielded better angular accuracy, when compared to bone-supported guides ${ }^{45}$.

A systematic review and meta-analysis was conducted in 2018 including 34 articles, to determine which guide type the literature found to be most accurate ${ }^{49}$. However, the results were completely divided with half of the articles saying toothsupported guides were more accurate and the other half determining mucosa-supported guides were best ${ }^{49}$. Therefore, a consensus could not be reached within the literature regarding implant placement accuracy according to guide type.

In regards to some of the other surgical variables, the literature determined flapless to be more accurate than a flapped approach ${ }^{20}$. The flapless approach showed smaller angular deviations as well as an overall improved accuracy of implant placement ${ }^{20}$. Also, the use of fixation screws significantly improved angular accuracy regardless of the surgical guide type $\mathrm{e}^{20 ; 46}$. Unfortunately, there is less consistent information when assessing positioning in the maxillary or mandibular arch, and when evaluating partially versus fully guided implant placement.

Two in vivo studies evaluated guided implant placement in both the maxillary and mandibular arches. Both found the angular accuracy to be significantly improved in the maxillary $\operatorname{arch}^{12 ; 45}$. They concluded that an implant is more likely to follow the path of the osteotomy, when the bone density is lower and the surgeon is met with less 
resistance $^{12 ; 44 ; 45}$. However, a recent meta-analysis determined that the mandible has better angular accuracy than the maxilla ${ }^{20}$. They postulated that this could be due to the difference in ridge shape ${ }^{20}$.

The literature does seem to agree that fully guided results are typically better than partially guided results; yet, they are divided when assessing if the difference is clinically significant. A study by Kuhl et al placed 38 implants in cadaver mandibles using SLA guides, to compare the difference between partially and fully guided implant placement ${ }^{13}$. While the fully guided positioning showed a trend towards increased accuracy, statistically significant differences were not found ${ }^{13}$. A meta-analysis however, found that fully guided placements resulted in significantly better implant angulation and reduced apical error, compared to partially guided implant placements ${ }^{20}$. It is worth noting, that of the 14 articles included in the meta-analysis, only two discussed partially versus fully guided placement ${ }^{20}$.

Another contributing factor to surgical guide inaccuracies is human error ${ }^{12 ; 39 ; 40}$. It can be challenging to properly position the guide due to clinical factors ${ }^{12 ; 39}$. When reflecting a flap, it is key to make sure the tissue does not prevent full seating of the surgical guide ${ }^{12}$. Furthermore, mucosa-supported guides can be tricky due the tissue's resiliency ${ }^{12}$. Also, the use of fixation screws is optimized when three screws can be utilized in a tripod formation (which is not always possible) ${ }^{12}$. Care should be taken to ensure that the guide is appropriately positioned prior to engaging the fixation screws. Additional patient specific variables include limited mouth opening and location of the implant ${ }^{12 ; 39}$. It can be challenging to place all the components required for a guided surgery in the posterior sextants, especially when inter-occlusal space is limited ${ }^{12 ; 39}$. Despite these challenges, the literature still indicates that SLA guided placement can compensate for lack of experience to yield superior results to free-handed placement ${ }^{40}$.

This leads to a discussion about the level of accuracy clinicians can expect from implant placement with the aid of on an SLA surgical guide. Several of the aforementioned studies list statistics regarding their implant placement accuracy. However, it can be difficult to make sense of all the data and put the information into 
perspective regarding the various study types. Therefore, the numbers to be emphasized are from two meta-analysis studies that were conducted within the last year. Zhou et al found the following average deviations: overall offset at the implant base was $1.25 \mathrm{~mm}$, overall offset at the apex was $1.57 \mathrm{~mm}$, lateral error at the implant base was $1.05 \mathrm{~mm}$, lateral error at the implant apex was $0.91 \mathrm{~mm}$, depth at the implant base was $0.64 \mathrm{~mm}$, depth at the implant apex was $1.24 \mathrm{~mm}$, and angular deviation was $4.1^{\circ 20}$. Bover-Ramos et al made the following safety distance recommendations based on the findings of their study: $1.7 \mathrm{~mm}$ in the apical horizontal direction, $1.0 \mathrm{~mm}$ in the vertical direction, and allow for $4.7^{\circ}$ of angular deviation ${ }^{49}$. This study also warns that these values may be slightly more accurate than can be expected in a clinical setting, since in vitro studies were included in their analysis ${ }^{20 ; 49}$.

\section{Domestic Pig as an Animal Model}

Animal models are of great value, since they provide a close degree of similarity to the human situation ${ }^{50 ; 51}$. This offers a superior level of significance to the data, compared to an in vitro study ${ }^{51}$. Rats and rabbits are some of the smaller species that have been used in dental research ${ }^{52}$. When focusing on implant studies, these animals usually require use of a long bone, such as a tibia or femur ${ }^{52}$. One big disadvantage is that these bones are formed by endochondral ossification, while bone in the maxilla and mandible is formed by intramembranous ossification ${ }^{52}$. Therefore, using a large animal model for this area of study is of great importance ${ }^{50}$. Some examples of suitable large animal models include monkeys, dogs, sheep, poultry, cows, and pigs/mini-pigs ${ }^{51 ; 52}$.

When focusing on research limited to dentistry, the ideal models are quickly narrowed to dogs and pigs/minipigs ${ }^{51 ; 53}$. Dog bone composition most closely resembles that of humans ${ }^{51}$. However, gaining ethical approval for this animal model can be challenging and the cost is typically high as well ${ }^{51 ; 52}$. When it comes to bone density measurements, both dogs and pigs most closely resemble the human condition ${ }^{51}$. Pigs also show similarities to human bone in regards to genetics, anatomy, morphology, and physiology $y^{53 ; 54}$. Another advantage of this model, is the ease of using medical imaging 
technologies as a diagnostic tool for research ${ }^{53}$. There are two main models used: the domestic pig and miniature pigs (mini-pigs $)^{53}$.

The use of pigs and miniature pigs has an established history as a dental model for research $^{50 ; 52 ; 54-61}$. Several implant studies have selected pigs to evaluate the biocompatibility of materials (zirconia vs titanium implants), osseointegration, bone-toimplant contact, and even the impact of certain diseases (such as MRONJ) ${ }^{52 ; 55-58 ; 61}$. This model is not limited only to implant research. It also includes assessment of soft tissue augmentation and comparison of bone density ${ }^{57 ; 59}$. Porcine specimens have been shown to be reliable in predicting fluoride-induced bone mass changes as well ${ }^{57}$. Furthermore, research surrounding pig enamel mineralization has led to great discoveries in understanding human amelogenesis ${ }^{53 ; 60}$.

The tooth structure of pigs/mini-pigs resembles that of humans more than any other species ${ }^{53}$. They share certain features of the human dentition, such as the bunodont shape of molars and brachydont size of teeth ${ }^{60 ; 62}$. This refers to the rounded cusps of molars and the shortened crown height of the dentition ${ }^{60}$. However, pig molars are typically more oblong in shape and have additional cusps (typically 5-6) with extra grooves, fossae, and eminences in the occlusal anatomy ${ }^{62}$. Also, their initial set of deciduous teeth have usually completed eruption between 6 and 8 months of age, while their permanent teeth take from 4 months to 2 years to finish erupting ${ }^{60 ; 62}$. The pig mandible can present some challenges as a model, due to the superficial position of a much larger inferior alveolar canal and the tilted position of the canines ${ }^{60}$.

The model used in this study consisted of six month old domestic pigs. At this phase of development, there was mainly a deciduous dentition present. The permanent dentition was developing within the jaw at this time as well, so care was taken to avoid the large inferior alveolar canal, tilted canine, and developing tooth buds when planning implant positions. 


\section{Chapter III: Materials and Methods}

\section{Preparation of the Experimental Model}

Pig jaws were collected from a local farm to be prepared for experimental use. The tongue, muscles, and extraneous soft tissues were removed, preserving only the mucosa surrounding the teeth and the edentulous ridge span (Figure 1 A). Each side was cut with a hack-saw to a dimension less than $10 \mathrm{~cm}$ in length, attempting to preserve as much of the anterior edentulous ridge as possible (Figure $1 \mathrm{~B}$ ). This measurement was required for the specimen to fit the maximum field of view of the CBCT. The jaws were then sectioned at the midline into right and left segments (Figure $2 \mathrm{~A}-\mathrm{C}$ ).
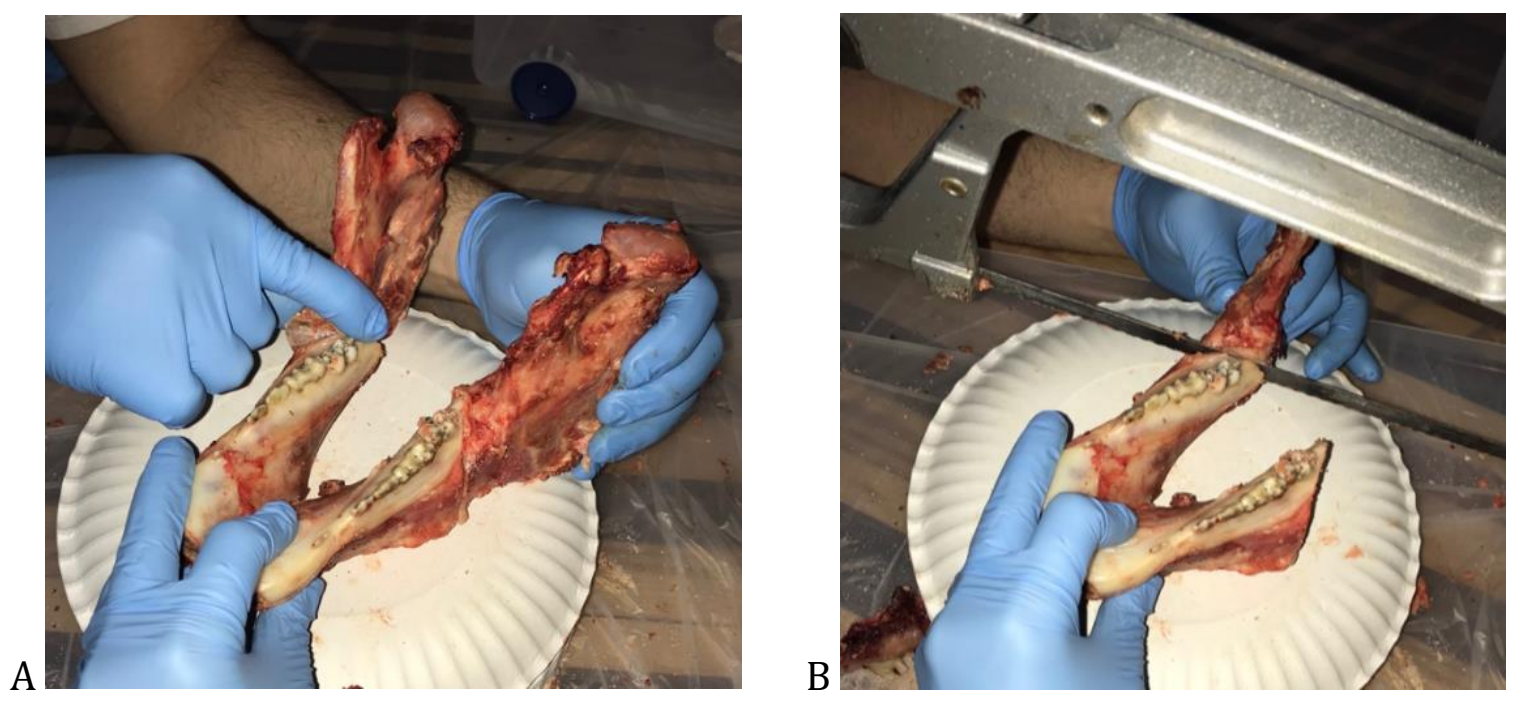

Figure 1 (A and B). Reduction in size of specimen: (A) Pig jaw after removal of tongue and dissection of surrounding muscles. (B) Sectioning of the posterior portion to provide a $10 \mathrm{~cm}$ length specimen. 

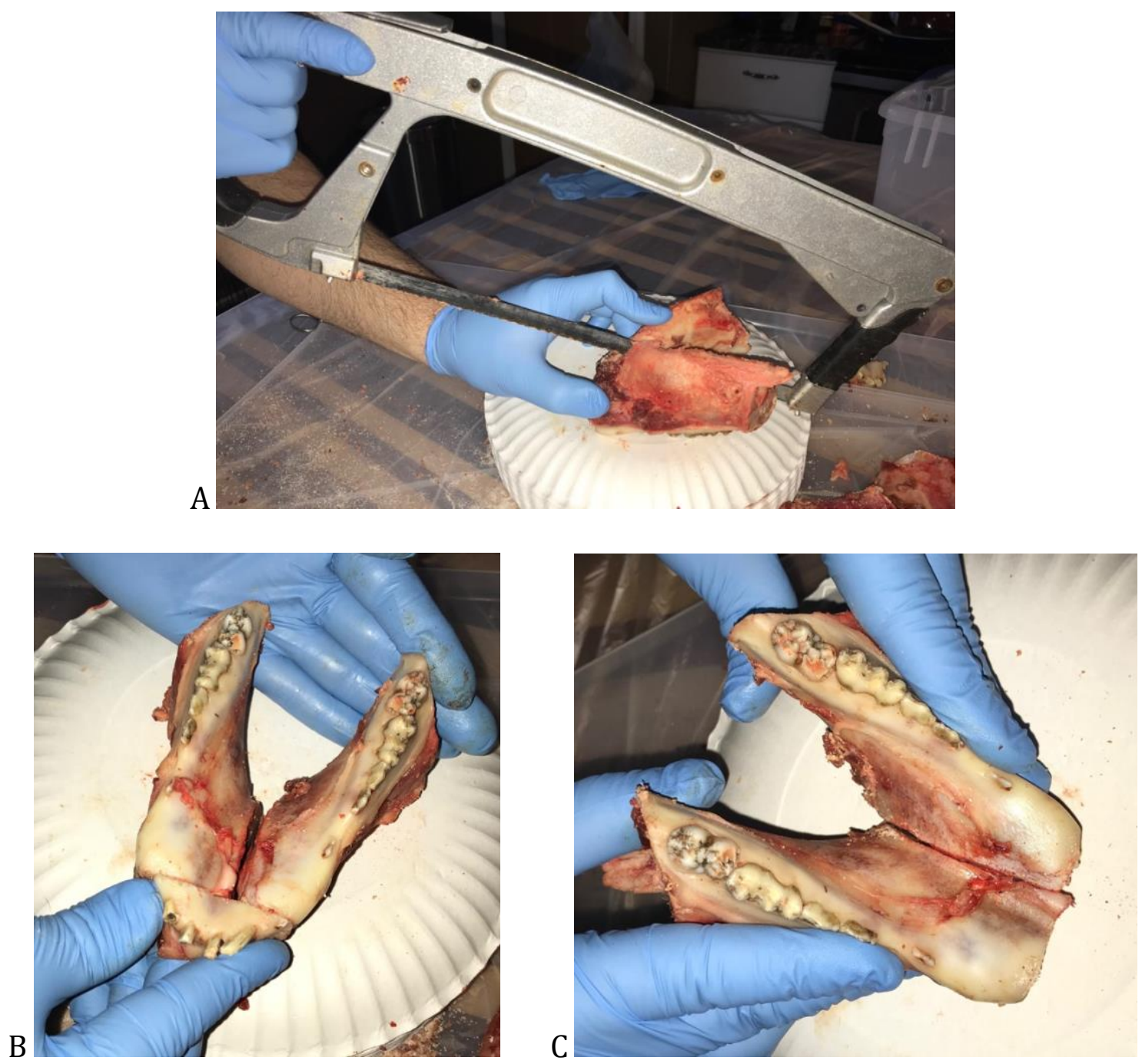

Figure 2 A-C. Preparation of the pig jaws: (A) The jaws were sectioned at the midline.

(B and C) The two specimens are shown, consisting of a right and left segment of one pig jaw. 


\section{Materials}

A rigid putty, Sil-Tech Super, was used to stabilize the jaws in an upright position (Ivoclar Vivadent Inc., Amherst, NY). The Carestream CS 9300-1 CBCT (Carestream Dental LLC, Atlanta, Georgia) was used, as well as the Straumann CARES Intraoral Scanner (Straumann International Headquarters, Basel, Switzerland). This data was loaded onto the coDiagnostiX software (Dentalwings, Montreal, Canada), which allowed printing of a surgical guide with the FormLabs Form 2 stereolithography (SLA) printer (FormLabs Inc., Somerville, MA). A clear UV-cured methacrylate photopolymerizing resin material (Dental SG; Formlabs Inc.) was used to print the surgical guides and the FormLabs Form Cure oven was required for curing the guides (Formlabs, Somerville, MA). The guides were then adjusted with Brasseler acrylic burs (Brasseler USA, Savannah, GA) and sterilized in the Ritter M11 UltraClave autoclave (Midmark Corporation, Dayton, $\mathrm{OH})$.

Twenty Straumann implants were used, including ten BLT implants (6 SLActive and 4 SLA) and ten BL implants (6 SLActive and 4 SLA). All implants were $4.1 \mathrm{~mm}$ in diameter and all but one implant was $12 \mathrm{~mm}$ in length. One BLT SLActive implant was $10 \mathrm{~mm}$ in length. Surgical guides had Straumann $\varnothing 5 \mathrm{~mm}$ metal sleeves (Straumann USA, LLC., Andover, MA). Both BL and BLT guided surgical cassettes were used in conjunction with a Zimmer Biomet surgical drill system (W\&H Dentalwerk GmbH, Burmoos, Austria) and a W\&H WS-75 L surgical contra-angle handpiece (W\&H Sterilization S.r.l., Brusaporto, Italy). Occlude aerosol indicator spray was used to aid in seating the guides (Pascal Company Inc., Bellevue, WA). Also, Straumann CARES RC Mono Scanbodies were required for post-operative scanning (Straumann USA, LLC., Andover, MA).

Treatment evaluation software was made available through the coDiagnostiX (Dentalwings, Montreal, Canada) software by Straumann. Information regarding the length of the surgical guide and distances from the tooth support, were measured using a Bolight digital caliper. 


\section{Protocol}

A custom platform was fabricated to appropriately position the model within the field of view of the CBCT. A rigid putty was also used to orient the sectioned jaw so the occlusal table was nearly level and the teeth/implant sites were centered (using the laser line as an aid). Each specimen was scanned using the Carestream CS 9300-1 CBCT Unit set to the following parameters: FoV $=10 \mathrm{~cm}$ X $10 \mathrm{~cm}$, Voxel Size $=300$, adult patient, $\mathrm{kV}=90, \mathrm{~mA}=4.0, \mathrm{~S}=8.00$, and $\mathrm{mGycm}^{2}=685$.

The jaws were then scanned with the Straumann CARES intraoral scanner, using a powder technique. The STL files were exported using a flash-drive.

The DICOM files that were reconfigured in the MIPACs software, were then transferred to coDiagnostiX using CS 3D Imaging to upload the files. First, a proper orientation of the sectioned jaw was achieved in sagittal, axial, and coronal views. Then, a curve for cross-sectional viewing was created, following the central fossa of the teeth and remaining centered along the edentulous span planned for implant positioning. Next, the respective STL file of the specimen's dentition and surgical site were aligned to the CBCT using prominent landmarks.

Once an acceptable registration was achieved, implant planning began. The first implant (A) was positioned adjacent to the tooth support, while a second implant (B) was planned further from the tooth support across an edentulous span. Care was taken to position the implant fully in native bone, avoiding growing tooth germs.

The sleeves and heights were selected, using only $5 \mathrm{~mm}$ diameter by $5 \mathrm{~mm}$ height sleeves for the positioning of $4.1 \mathrm{~mm}$ Straumann implants as indicated in Table 1.

Table 1. Straumann $4.1 \mathrm{~mm}$ diameter implant descriptions according to guide number.

\begin{tabular}{|c|c|c|c|c|c|c|c|c|c|c|c|}
\hline $\begin{array}{c}\text { Guide } \\
\text { Number }\end{array}$ & 1 & 2 & 3 & 4 & 5 & 6 & 7 & \multicolumn{2}{|c|}{8} & 9 & 10 \\
\hline $\begin{array}{c}\text { Implant } \\
\text { Type }\end{array}$ & BLT & BL & BL & BL & BLT & BLT & BLT & \multicolumn{2}{|c|}{ BLT } & $\mathrm{BL}$ & $\mathrm{BL}$ \\
\hline $\begin{array}{l}\text { Implant } \\
\text { Surface }\end{array}$ & SLA & SLActive & SLActive & SLActive & SLA & SLActive & SLActive & \multicolumn{2}{|c|}{ SLActive } & SLA & SLA \\
\hline $\begin{array}{c}\text { Implant } \\
\text { length }\end{array}$ & 12 & 12 & 12 & 12 & 12 & 12 & 12 & $A=12$ & $\mathrm{~B}=10$ & 12 & 12 \\
\hline
\end{tabular}


The guide was designed with appropriate angulation for passive seating, through elimination of undercuts (Figure 3). Sufficient coverage was outlined around the scanned teeth from the IOS STL file (Figure $4 \mathrm{~A}$ and C). The guide thickness was set to $3 \mathrm{~mm}$, and offset to teeth was $0.2 \mathrm{~mm}$. A rigid strut was used to connect the sleeves for both position A and B implants, rather than designing mucosal coverage (Figure $4 \mathrm{~B}$ ). Two inspection windows were added in similar positions for each guide to aid in verification of proper seating. Labels were embossed on the printed surgical guide, which indicated the guide number, implant type, and implant length (Figure 4 B and D). The surgical guides were then oriented on the 3-D printer platform, to prevent the supports from contacting the intaglio surface of the guide. Two separate cycles of 3-D printing were required to print all ten guides. The data was sent to a FormLabs Form 2 SLA printer, to be printed with the photo-polymerizing resin material. Upon completion, the guides were soaked twice for 10 minutes each in separate baths of $91 \%$ isopropyl alcohol. Afterwards, the guides were given 30 minutes to air dry before inserting the guides into the FormLabs Form Cure oven for final curing for an additional 30 minutes at $60^{\circ} \mathrm{C}$.

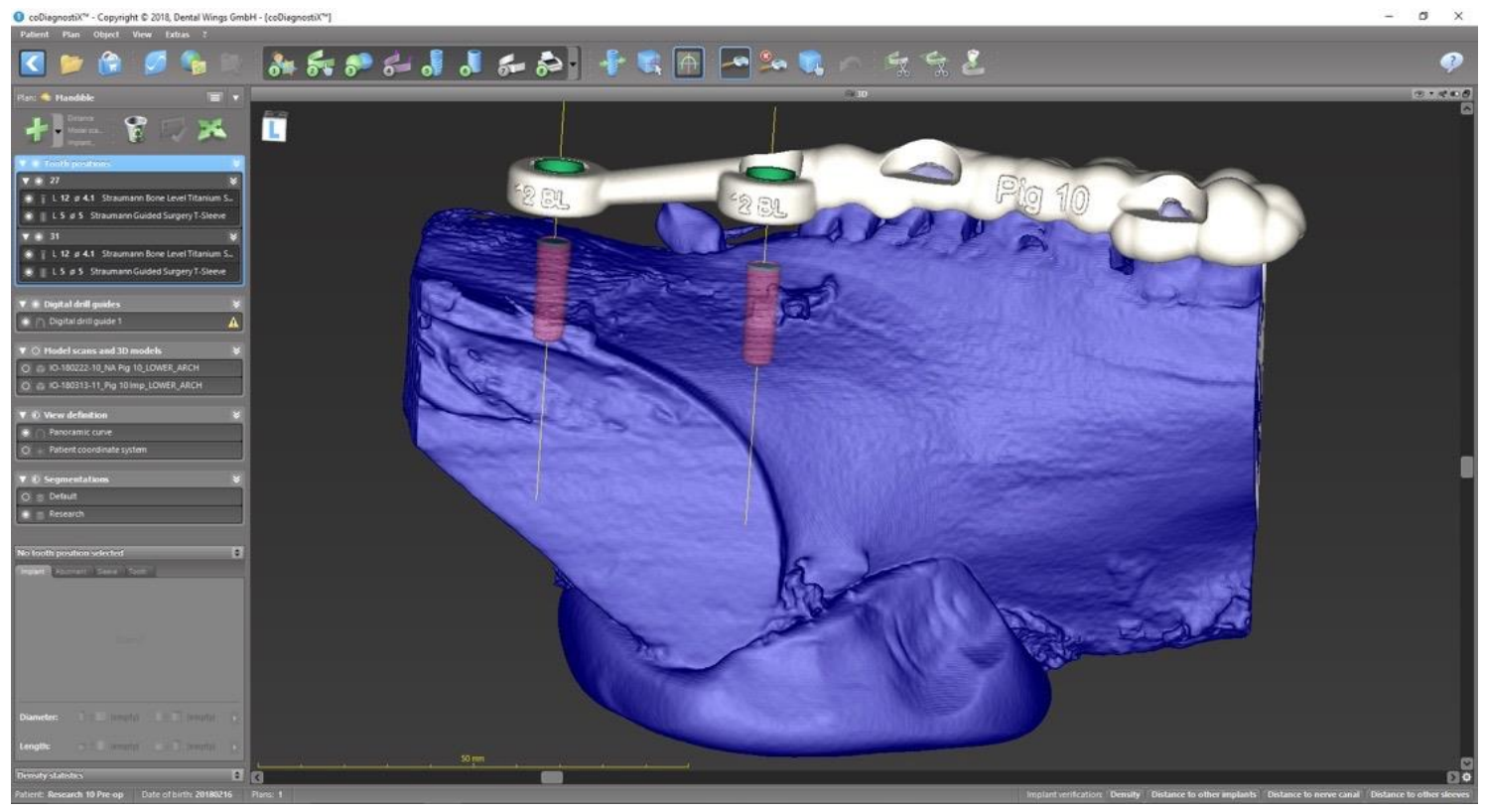

Figure 3. Finalized digital design of surgical guide. 

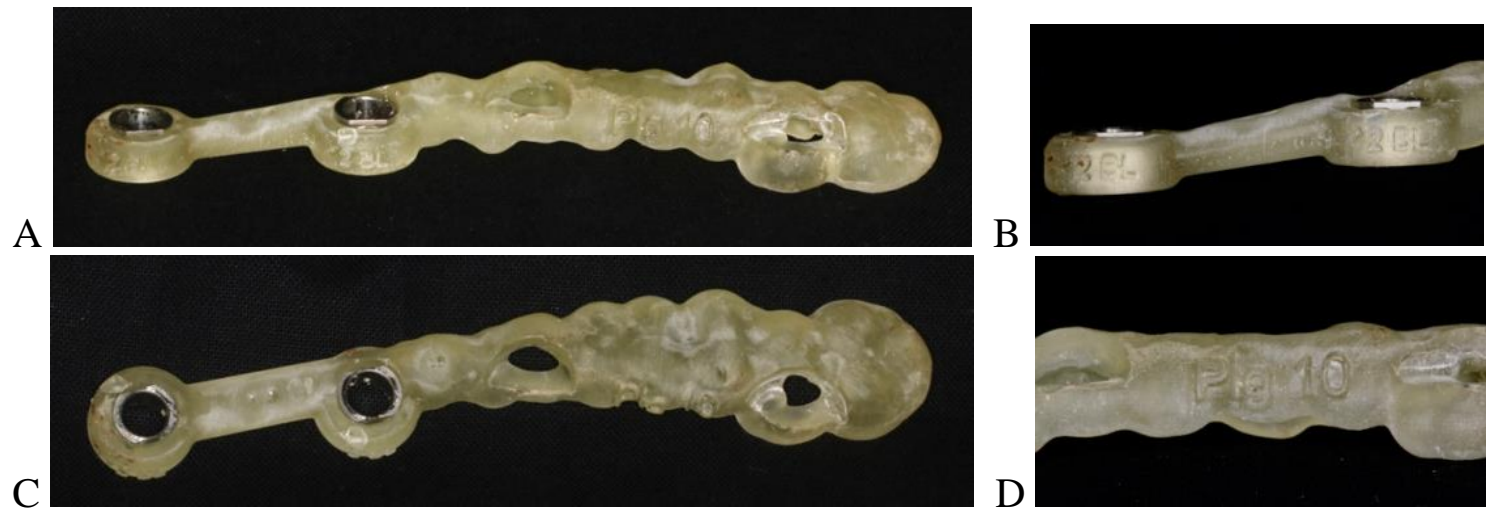

C

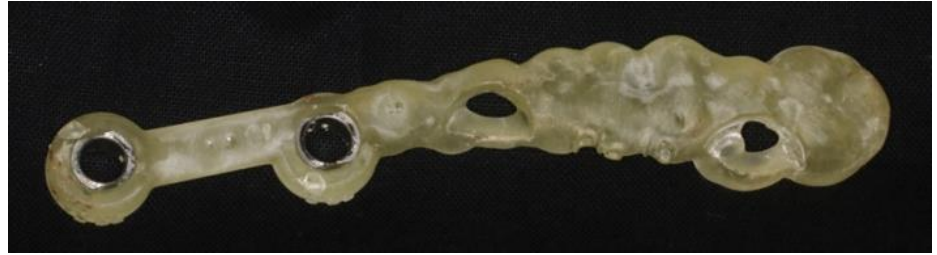

Figure 4 A-D. Surgical guide: (A) Lateral view. (B) Embossed implant types and lengths. (C) Occlusal view. (D) Embossed guide number.

The guides were sectioned and trimmed using a Brasseler serrated disc and flush cutters provided by FormLabs. They were then placed in the autoclave for sterilization, as indicated for patient use. Guide 3 fractured upon removal from the autoclave. However, the pieces were easily re-approximated and reinforced with Esthet-X HD flowable composite resin for use in the study (DENTSPLY International Inc., Australia).

The guides were tried in for each specimen, and adjusted as necessary to fully seat the guide with the aid of Occlude. The need for adjustment was documented (Table 2).

Implants were placed by the same clinician, following Straumann surgical protocols with the exception of utilizing irrigation. All sites were first decorticated with the $3.5 \mathrm{~mm}$ milling cutter at $500 \mathrm{RPM}$. The osteotomy was sequentially prepared using the sleeve indicated by the planned protocols in the following order: $2.2 \mathrm{~mm}$ pilot drill at 800 RPM, $2.8 \mathrm{~mm}$ drill at $600 \mathrm{RPM}$, and the $3.5 \mathrm{~mm}$ drill at $500 \mathrm{RPM}$. All sites were then prepared with the profile drill with the appropriate C-Handle at 300 RPMs. The sites planned for BL implants were tapped to full depth, at a maximum of $45 \mathrm{Ncm}^{2}$. Each implant was placed "free-handed" with the surgical hand-piece at $35 \mathrm{Ncm}^{2}$ until the primary stability was achieved, or full seating was observed. The final insertion torque value was documented (Table 2). 
The jaws were then post-operatively scanned in the CBCT, using the same settings and oriented by positioning marks made in the putties with a marker to match the laser lines. A post-operative intraoral scan was also performed for each specimen after hand-tightening scan bodies onto each implant, with the Straumann CARES IOS scanner. This allowed two methods to be used for post-operative assessment of the accuracy of implant placement.

A treatment evaluation software was made available by Dental Wings to assess the accuracy of the final implant positioning. The software allowed each post-operative data-set to be compared to the planned implant positioning, which provided the following deviations: angle, 3D offset, distal, vestibular, and apical errors in positioning (for both the implant platform and apex) (Figure 6 B).

The CBCT post-operative file was imported into coDiagnostiX using the same method described above for the pre-operative scan. The scan was then segmented, adjusting the gray value to most clearly visualize the teeth and labeled "Research". The same parameters were then used to segment the pre-operative scan. This facilitated seamless overlapping of the images for the post-operative comparison (Figure $5 \mathrm{~A}$ and B). A "planned implant" outline was then manually manipulated to be centered within the radiopaque implant position, as viewed in the sagittal, axial, and coronal planes (Figure $5 \mathrm{C}$ ). The software then calculated the error values, which were recorded.

The IOS comparison was more straightforward. The scan was loaded into the pre-operative file and aligned with the segmented pre-operative scan. The software then calculated the trajectory and depth of the implant based on the platform of the scan body (Figure $6 \mathrm{~A}$ ). This information was used to compare the planned positioning versus the clinical positioning indicated by the scan bodies (Figure $6 \mathrm{~B}$ ). The results were documented for analysis. 

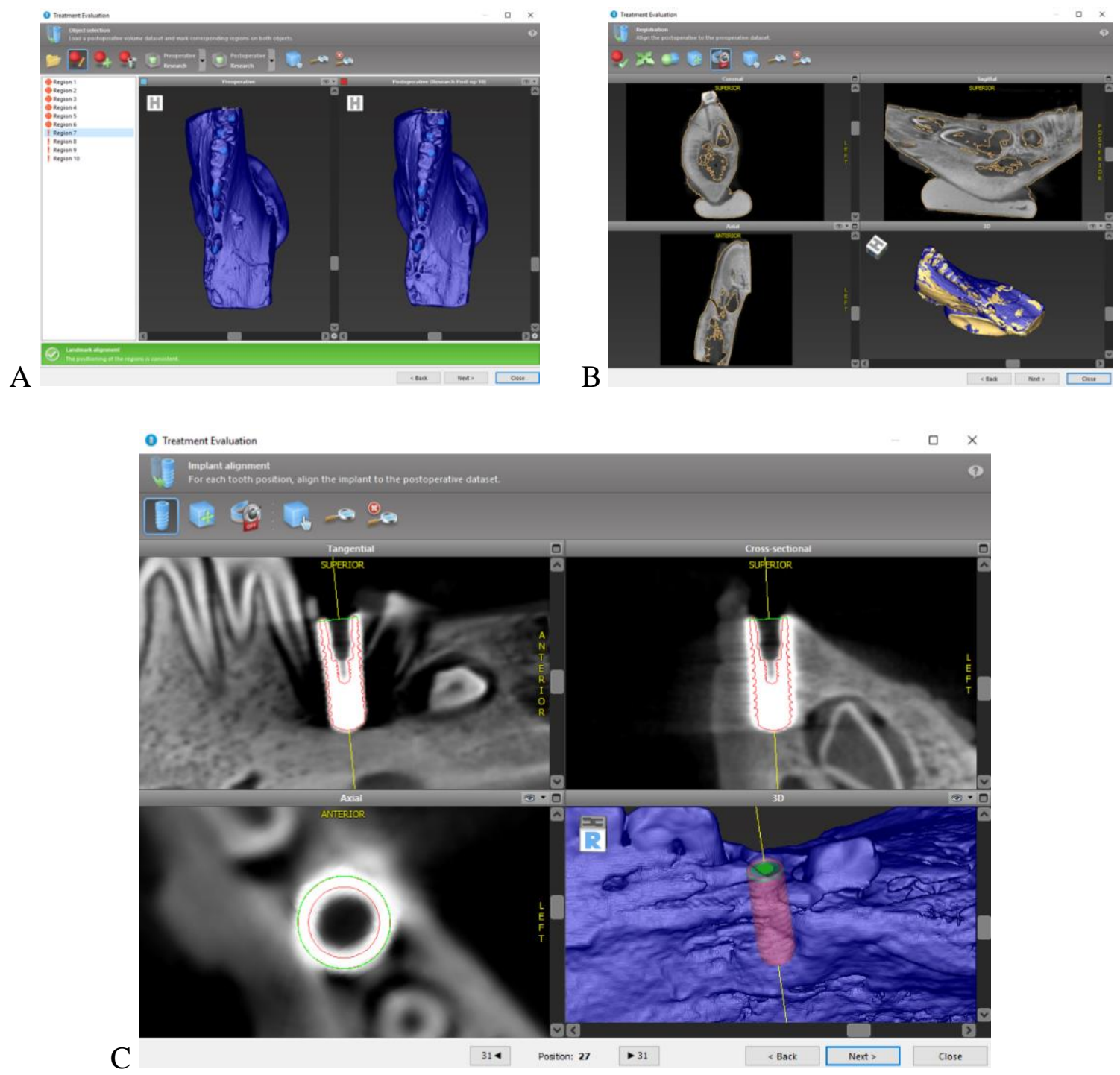

Figure 5 A-C. CBCT treatment evaluation: (A) Alignment of pre-operative and postoperative segmentations. (B) Merging of the scans. (C) Manual positioning of the digital implant within the confines of the radiopaque implant from the CBCT.
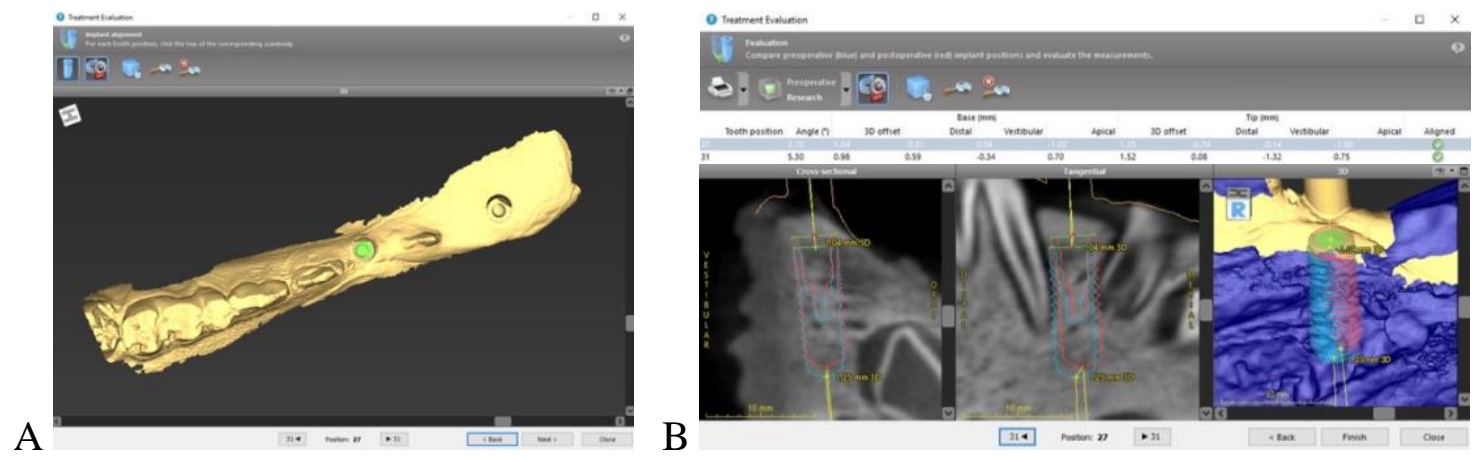

Figure $6 \mathrm{~A}$ and B. IOS treatment evaluation: (A) Selecting the platform of the scan body for Implant A. (B) Treatment evaluation data. 


\section{Statistical Analysis}

The surgical information, regarding implant type and length, was documented for each surgical guide and implant position (Table 2). The insertion torque was also recorded for each implant placed using the Straumann torque wrench (Table 2). Whether or not the guide required adjustment to fully seat was noted as well (Table 2). The distance from the intaglio position of termination of tooth support to the middle of the guide sleeve was measured using a caliper (accurate to $0.01 \mathrm{~mm}$ ). The total length of the guide was also measured and recorded (Table 2).

The raw data from the software indicated deviations at both the implant platform (base or coronal portion) and the implant apex (tip). For both of these sites, the software calculated the deviation in the distal, vestibular, and apical direction. The distal value denoted a distal discrepancy when positive, and a mesial discrepancy when negative. The vestibular value indicated a buccal deviation when positive, and a lingual deviation when negative. The apical value showed the implant was positioned beyond the planned depth when positive, and short of the intended depth when negative. The total 3D offset was also listed, which was the calculated hypotenuse of the three points created by the distal, vestibular, and apical data. The total angular discrepancy was listed in degrees. All the data was entered into a Microsoft Excel spreadsheet and overall averages were calculated for both CBCT and IOS data sets (Table 3 and 4).

The entirety of the data was analyzed to determine average discrepancies and $95 \%$ confidence intervals at the implant base and tip in each direction, and average angulation error. A Student t-test was then conducted to find the t-ratio and $\mathrm{p}$-value $(<0.05)$ to determine if significant differences were observed.

The CBCT data and IOS data were compared by performing a mixed-effects ANOVA, to see if the two methods for determining the deviations were significantly different.

A series of Student t-tests were then conducted to compare additional variables: implant position (A vs B), implant type (BL vs BLT), and if adjustments were required 
(yes vs no). The mean differences were calculated for each, as well as the t-ratio and pvalue to evaluate if there was significance among the deviations. A one-way ANOVA was then performed for the results showing significance. 


\section{Chapter IV: Results and Discussion}

\section{RESULTS}

The raw data from the surgical procedures can be seen in Table 2. Note that primary stability was achieved in all implants placed in position A site, compared to lower torque values found in four of the ten implant B sites. Half of the surgical guides required adjustment to fully seat, as visualized through the inspection windows. The average distance from the rigid support for implant A was $5.67 \mathrm{~mm}$, and implant B was $28.57 \mathrm{~mm}$. The average total length of the guides was $82.20 \mathrm{~mm}$.

Table 2. Surgical guide and implant placement data.

\begin{tabular}{|c|c|c|c|c|c|c|c|c|c|c|c|}
\hline \multicolumn{2}{|c|}{ Implant Guide Number } & $\mathbf{1}$ & $\mathbf{2}$ & $\mathbf{3}$ & $\mathbf{4}$ & $\mathbf{5}$ & $\mathbf{6}$ & $\mathbf{7}$ & $\mathbf{8}$ & $\mathbf{9}$ & $\mathbf{1 0}$ \\
\hline Implant Type & & BLT & BL & BL & BL & BLT & BLT & BLT & BLT & BL & BL \\
\hline \multirow{2}{*}{ Insertion Torque (Ncm2) } & A & 35 & 35 & 35 & 35 & 35 & 35 & 35 & 35 & 35 & 35 \\
\cline { 2 - 12 } & B & 35 & 35 & $<15$ & 20 & 35 & 35 & $<15$ & 35 & $<15$ & 35 \\
\hline Adjustments to Guide & & No & No & Yes & Yes & Yes & No & No & Yes & Yes & No \\
\hline \multirow{2}{*}{ Distance from Rigid Support (mm) } & A & 7.84 & 6.09 & 5.32 & 5.03 & 5.90 & 4.27 & 7.36 & 6.41 & 4.67 & 3.79 \\
\cline { 2 - 11 } & B & 27.57 & 28.01 & 27.36 & 27.42 & 22.36 & 23.52 & 36.84 & 39.98 & 24.01 & 28.67 \\
\hline Total Length of Guide (mm) & & 74.22 & 69.76 & 90.32 & 90.83 & 74.42 & 83.59 & 94.97 & 80.22 & 70.68 & 93.01 \\
\hline $\begin{array}{c}\text { Distance b/t base of the sleeve \& } \\
\text { implant platform (mm) }\end{array}$ & $\mathrm{A}$ & 6 & 6 & 6 & 6 & 6 & 6 & 6 & 6 & 6 & 6 \\
\cline { 2 - 11 } & $\mathrm{B}$ & 4 & 4 & 4 & 4 & 6 & 6 & 4 & 6 & 4 & 4 \\
\hline
\end{tabular}

The data collected from the Dental Wings treatment evaluation software is listed in Table 3 for the CBCT post-operative comparison and Table 4 for the IOS postoperative comparison. The mixed-analysis ANOVA concluded that there was no difference in the deviations gathered using each method. The data was then combined to calculate average discrepancies and perform a Student t-test (Table 5). There were significant deviations from zero at both the base and tip in the distal direction and for the 3D offset. The average discrepancy of the 3D offset at the base was $1.43 \mathrm{~mm}$ and a mesial average deviation of $0.18 \mathrm{~mm}$ was found. The mean 3D offset at the tip was 2.04 $\mathrm{mm}$ and there was an average mesial error of $0.71 \mathrm{~mm}$. The angular deviation was also found to be significant, with a mean error of $5.17^{\circ}$. The vestibular and apical errors were not found to be significant either at the base or the tip of the implant. 
Table 3. CBCT deviations of implant positioning.

\begin{tabular}{|c|c|c|c|c|c|c|c|c|c|}
\hline & \multicolumn{3}{|c|}{ Base or Coronal (mm) } & \multicolumn{3}{c|}{ Tip or Apex (mm) } & \\
\hline $\begin{array}{c}\text { Implant } \\
\text { Position }\end{array}$ & $\begin{array}{c}\text { 3D } \\
\text { offset }\end{array}$ & Distal & Vestibular & Apical & $\begin{array}{c}\text { 3D } \\
\text { offset }\end{array}$ & Distal & Vestibular & Apical & $\begin{array}{c}\text { Angle } \\
\text { (degrees) }\end{array}$ \\
\hline 1A & 0.86 & 0.33 & 0.65 & -0.47 & 0.87 & 0.67 & 0.31 & -0.46 & 2.30 \\
\hline 1B & 1.32 & 0.23 & 0.94 & 0.91 & 1.50 & 0.86 & 0.80 & 0.93 & 3.10 \\
\hline 2A & 0.77 & -0.22 & 0.61 & -0.42 & 1.89 & -1.32 & 1.30 & -0.35 & 6.20 \\
\hline 2B & 1.29 & -0.07 & -0.39 & 1.23 & 2.01 & -1.04 & 1.06 & 1.35 & 8.30 \\
\hline 3A & 1.61 & -0.36 & -1.41 & 0.69 & 2.82 & -0.15 & -2.17 & 0.76 & 6.30 \\
\hline 3B & 1.65 & 0.68 & -1.50 & -0.04 & 2.82 & 2.55 & -1.19 & 0.11 & 9.10 \\
\hline 4A & 1.04 & 0.13 & 0.97 & -0.35 & 2.13 & -0.66 & 2.01 & -0.28 & 6.20 \\
\hline 4B & 1.03 & 0.14 & -0.73 & 0.72 & 0.87 & -0.42 & -0.13 & 0.75 & 3.90 \\
\hline 5A & 0.97 & 0.07 & 0.89 & -0.39 & 2.18 & -0.61 & 2.07 & -0.31 & 6.50 \\
\hline 5B & 1.02 & 0.04 & -0.76 & 0.69 & 0.85 & -0.39 & -0.29 & 0.70 & 3.00 \\
\hline 6A & 2.00 & -0.61 & -0.15 & -1.90 & 2.41 & -1.40 & -0.61 & -1.87 & 4.40 \\
\hline 6B & 0.76 & -0.16 & -0.20 & -0.72 & 1.39 & -1.20 & -0.20 & -0.68 & 5.00 \\
\hline 7A & 1.54 & -0.47 & 0.92 & -1.14 & 2.06 & -1.33 & 1.12 & -1.11 & 4.20 \\
\hline 7B & 1.73 & -1.47 & 0.85 & 0.36 & 2.77 & -2.73 & -0.08 & 0.46 & 7.50 \\
\hline 8A & 2.73 & -0.78 & -0.43 & -2.57 & 3.42 & -2.12 & -1.02 & -2.49 & 7.00 \\
\hline 8B & 1.03 & -0.74 & 0.35 & -0.62 & 2.24 & -2.03 & 0.80 & -0.53 & 7.80 \\
\hline 9A & 2.50 & -0.44 & 0.17 & -2.45 & 2.89 & -1.63 & 0.08 & -2.39 & 5.70 \\
\hline 9B & 1.37 & -0.27 & 0.63 & -1.19 & 2.22 & -1.90 & 0.43 & -1.07 & 7.80 \\
\hline 10A & 1.42 & -0.31 & 0.64 & -1.23 & 1.94 & -1.34 & 0.73 & -1.19 & 4.90 \\
\hline 10B & 0.79 & 0.02 & 0.33 & -0.71 & 1.30 & -1.13 & 0.01 & -0.65 & 5.70 \\
\hline Averages & $\mathbf{1 . 3 7}$ & $-\mathbf{0 . 2 1}$ & $\mathbf{0 . 1 2}$ & $-\mathbf{0 . 4 8}$ & $\mathbf{2 . 0 3}$ & $-\mathbf{- 0 . 8 7}$ & $\mathbf{0 . 2 5}$ & $-\mathbf{0 . 4 2}$ & $\mathbf{5 . 7 5}$ \\
\hline
\end{tabular}

Table 4. IOS deviations of implant positioning.

\begin{tabular}{|c|c|c|c|c|c|c|c|c|c|}
\hline & \multicolumn{3}{|c|}{ Base or Coronal $(\mathbf{m m})$} & \multicolumn{3}{c|}{ Tip or Apex (mm) } & \\
\hline $\begin{array}{c}\text { Implant } \\
\text { Position }\end{array}$ & $\begin{array}{c}\text { 3D } \\
\text { offset }\end{array}$ & Distal & Vestibular & Apical & $\begin{array}{c}\text { 3D } \\
\text { offset }\end{array}$ & Distal & Vestibular & Apical & $\begin{array}{c}\text { Angle } \\
\text { (degrees) }\end{array}$ \\
\hline 1A & 0.79 & -0.06 & 0.78 & -0.10 & 0.76 & 0.04 & 0.75 & -0.10 & 0.50 \\
\hline 1B & 2.00 & -0.37 & 0.91 & 1.75 & 2.11 & 0.12 & 1.15 & 1.76 & 2.60 \\
\hline 2A & 0.81 & -0.31 & 0.67 & -0.35 & 2.10 & -1.25 & 1.66 & -0.27 & 6.60 \\
\hline 2B & 1.89 & 0.08 & -0.08 & 1.89 & 2.56 & -0.56 & 1.49 & 2.01 & 8.10 \\
\hline 3A & 1.44 & -0.08 & -1.30 & 0.61 & 2.58 & 0.20 & -2.49 & 0.67 & 5.80 \\
\hline 3B & 1.32 & 0.59 & -1.17 & 0.19 & 2.54 & 2.41 & -0.75 & 0.33 & 9.00 \\
\hline 4A & 0.53 & 0.08 & 0.52 & 0.03 & 1.51 & -0.13 & 1.51 & 0.08 & 4.80 \\
\hline 4B & 2.31 & 0.23 & -1.32 & 1.88 & 2.24 & 0.02 & -1.21 & 1.88 & 1.10 \\
\hline 5A & 0.37 & 0.06 & 0.37 & -0.01 & 1.18 & -0.15 & 1.17 & 0.02 & 3.90 \\
\hline 5B & 2.29 & 0.15 & -1.43 & 1.78 & 2.33 & -0.13 & -1.49 & 1.79 & 1.40 \\
\hline 6A & 1.70 & -0.30 & -0.29 & -1.65 & 1.89 & -0.75 & -0.57 & -1.64 & 2.50 \\
\hline 6B & 0.40 & 0.38 & -0.12 & 0.06 & 0.40 & -0.38 & -0.11 & 0.09 & 3.60 \\
\hline 7A & 2.38 & -0.54 & 2.00 & -1.17 & 3.12 & -1.19 & 2.65 & -1.14 & 4.40 \\
\hline 7B & 2.65 & -1.95 & 1.66 & 0.71 & 3.72 & -3.39 & 1.33 & 0.80 & 7.10 \\
\hline 8A & 2.45 & -0.77 & 0.39 & -2.29 & 2.93 & -1.80 & 0.55 & -2.25 & 5.00 \\
\hline 8B & 0.81 & -0.05 & 0.74 & 0.33 & 1.85 & -0.51 & 1.74 & 0.39 & 6.30 \\
\hline 9A & 2.37 & -0.43 & 0.44 & -2.29 & 2.62 & -1.30 & 0.24 & -2.26 & 4.30 \\
\hline 9B & 1.30 & -0.11 & 1.18 & -0.54 & 1.88 & -1.50 & 1.03 & -0.46 & 6.70 \\
\hline 10A & 1.04 & -0.21 & 0.04 & -1.02 & 1.25 & -0.73 & -0.14 & -1.00 & 2.70 \\
\hline 10B & 0.98 & 0.59 & -0.34 & 0.70 & 1.52 & 0.07 & -1.32 & 0.75 & 5.30 \\
\hline Averages & 1.49 & -0.15 & $\mathbf{0 . 1 8}$ & $\mathbf{0 . 0 3}$ & $\mathbf{2 . 0 5}$ & $-\mathbf{0 . 5 5}$ & $\mathbf{0 . 3 6}$ & $\mathbf{0 . 0 7}$ & $\mathbf{4 . 5 9}$ \\
\hline
\end{tabular}


Table 5. Average discrepancies in each direction at the base and tip of implants, confidence intervals, Student's $t$-test values, and p-values.

\begin{tabular}{|c|c|c|c|c|c|c|}
\hline \multicolumn{2}{|c|}{ Direction } & $\begin{array}{c}\text { Lower 95\% } \\
\text { Mean }\end{array}$ & Mean & $\begin{array}{c}\text { Upper } 95 \% \\
\text { Mean }\end{array}$ & t-ratio & p-value \\
\hline \multirow{4}{*}{$\begin{array}{l}\bar{\xi} \\
\underline{\xi} \\
\tilde{d} \\
\tilde{D} \\
\infty\end{array}$} & 3D offset & 1.22 & 1.43 & 1.64 & 13.78 & $0.0000 *$ \\
\hline & Distal & -0.34 & -0.18 & -0.02 & -2.27 & $0.0287^{*}$ \\
\hline & Vestibular & -0.13 & 0.15 & 0.43 & 1.10 & 0.2780 \\
\hline & Apical & -0.61 & -0.23 & 0.15 & -1.21 & 0.2347 \\
\hline \multirow{4}{*}{$\begin{array}{l}\widehat{\xi} \\
\underline{\xi} \\
\stackrel{\varrho}{\models}\end{array}$} & 3D offset & 1.80 & 2.04 & 2.28 & 17.02 & 0.0000* \\
\hline & Distal & -1.07 & -0.71 & -0.34 & -3.88 & $0.0004^{*}$ \\
\hline & Vestibular & -0.07 & 0.31 & 0.68 & 1.63 & 0.1110 \\
\hline & Apical & -0.55 & -0.17 & 0.21 & -0.91 & 0.3681 \\
\hline \multicolumn{2}{|c|}{ Angle (degrees) } & 4.47 & 5.17 & 5.86 & 14.99 & 0.0000* \\
\hline
\end{tabular}

$*$ p-values $<0.05$ indicate significance (JMP/Pro Ver12, SAS Inst. Inc., Cary, NC).

Implant position A and B were compared using the Student's t-test (Table 6). A significant difference was found only in the apical direction at both the base and tip of the implant. Implant A was found to be positioned short of the planned position, while Implant B was positioned deeper than planned (Figure 7 and 8).

Table 6. Student's t-test comparing implant position A and B.

\begin{tabular}{|c|c|c|c|c|}
\hline \multicolumn{2}{|c|}{ Direction } & Difference & t-ratio & p-value \\
\hline \multirow{4}{*}{ 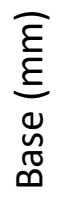 } & 3D offset & -0.07 & -0.33 & 0.7446 \\
\hline & Distal & 0.16 & 0.99 & 0.3307 \\
\hline & Vestibular & -0.35 & -1.27 & 0.2102 \\
\hline & Apical & 1.39 & 4.53 & $0.0001^{*}$ \\
\hline \multirow{4}{*}{$\begin{array}{l}\widehat{\underline{\varepsilon}} \\
\underline{\underline{E}} \\
\stackrel{\varrho}{ }\end{array}$} & 3D offset & -0.17 & -0.71 & 0.4819 \\
\hline & Distal & 0.28 & 0.77 & 0.4434 \\
\hline & Vestibular & -0.30 & -0.81 & 0.4242 \\
\hline & Apical & 1.41 & 4.63 & $0.0000^{*}$ \\
\hline \multicolumn{2}{|c|}{ Angle (degrees) } & 0.91 & 1.33 & 0.1902 \\
\hline
\end{tabular}

* p-values < 0.05 indicate significance (JMP/Pro Ver12, SAS Inst. Inc., Cary, NC). 


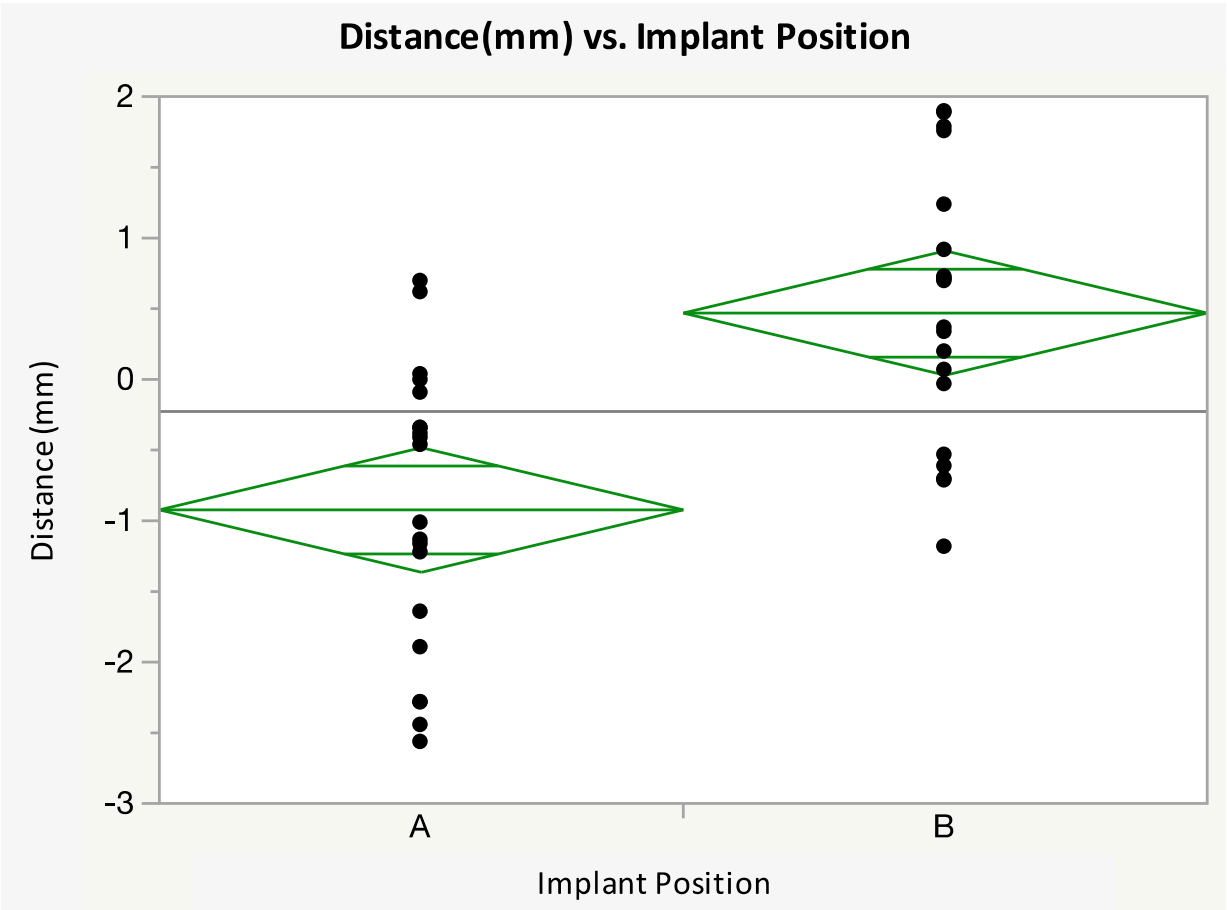

Figure 7. Difference in distance between position A and B in the Base - Apical direction (JMP/Pro Ver12, SAS Inst. Inc., Cary, NC).

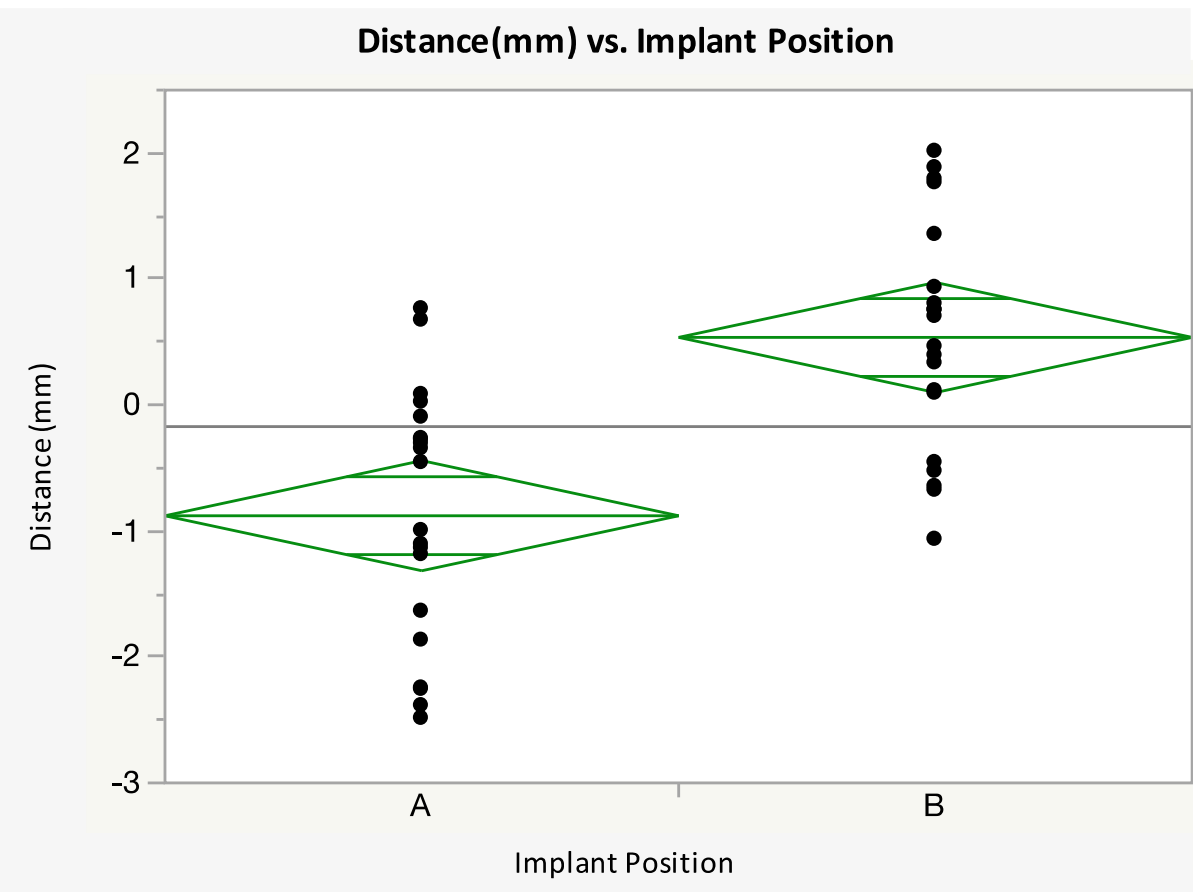

Figure 8. Difference in distance between position A and B in the Apex - Apical direction (JMP/Pro Ver12, SAS Inst. Inc., Cary, NC). 
A comparison was performed using the Student t-test for implant type as well (Table 7). A significant difference was found between BL and BLT implants at the base of the implant in the mesial/distal direction and in the overall angulation error. The BL implants were found have a minimal distal error, while BLT implants showed a larger distal error (Figure 9). However, BLT implants showed a lower angulation error, compared to BL implants which showed a higher angular discrepancy (Figure 10). The BL implants showed a trend towards a lingual error, compared to the BLT implants displaying positional errors towards the buccal (Figure 11).

Table 7. Student's t-test comparing implant type BL to BLT.

\begin{tabular}{|c|c|c|c|c|}
\hline \multicolumn{2}{|c|}{ Direction } & Difference & t-ratio & p-value \\
\hline \multirow{4}{*}{ 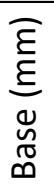 } & 3D offset & 0.12 & 0.56 & 0.5801 \\
\hline & Distal & -0.34 & -2.20 & $0.0337 *$ \\
\hline & Vestibular & 0.51 & 1.91 & 0.0643 \\
\hline & Apical & -0.19 & -0.50 & 0.6211 \\
\hline \multirow{4}{*}{$\begin{array}{l}\widehat{\underline{\xi}} \\
\underline{\xi} \\
\stackrel{\varrho}{\models}\end{array}$} & 3D offset & -0.09 & -0.35 & 0.7265 \\
\hline & Distal & -0.43 & -1.19 & 0.2420 \\
\hline & Vestibular & 0.40 & 1.06 & 0.2965 \\
\hline & Apical & -0.22 & -0.58 & 0.5656 \\
\hline \multicolumn{2}{|c|}{ Angle (degrees) } & -1.52 & -2.33 & $0.0253 *$ \\
\hline
\end{tabular}

* p-values < 0.05 indicate significance (JMP/Pro Ver12, SAS Inst. Inc., Cary, NC).

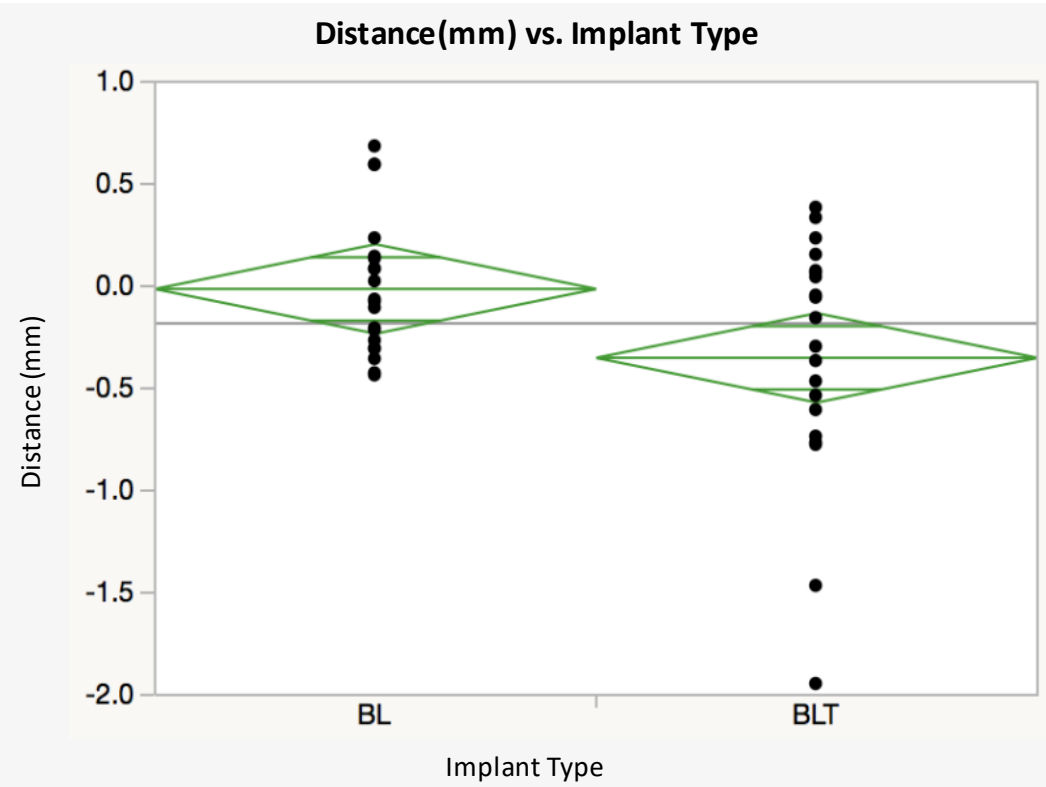

Figure 9. Difference between implant type BL and BLT in the Base - Distal direction (JMP/Pro Ver12, SAS Inst. Inc., Cary, NC). 


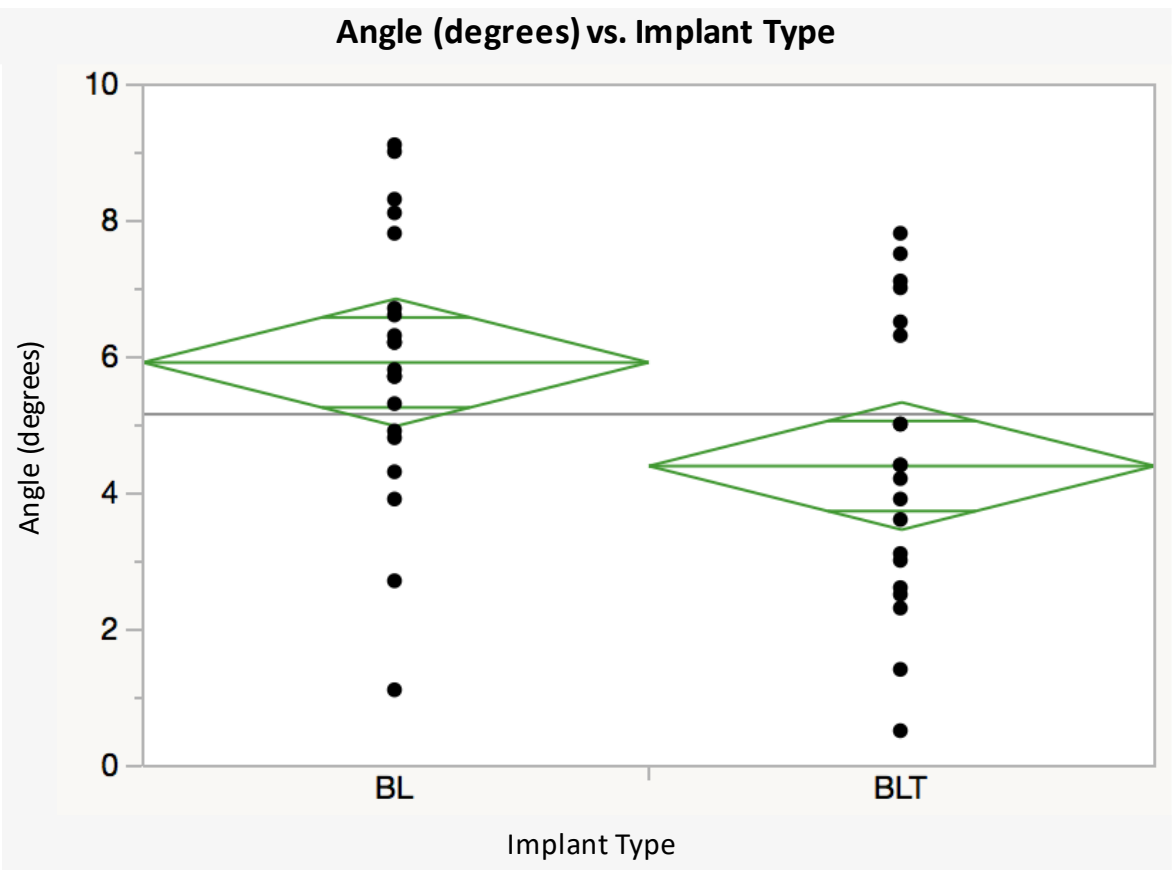

Figure 10. Difference in angulation between implant types BL and BLT (JMP/Pro Ver12, SAS Inst. Inc., Cary, NC).

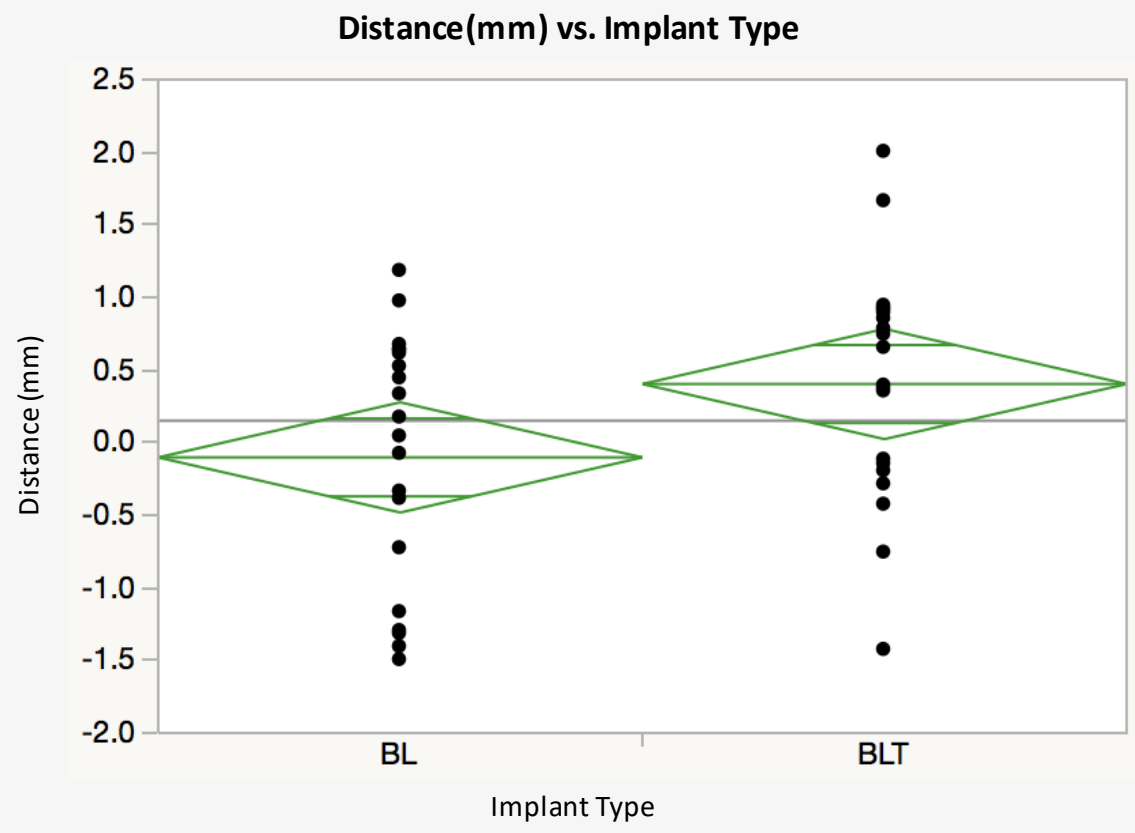

Figure 11. Difference between implant type BL and BLT in the Base - Vestibular direction (JMP/Pro Ver12, SAS Inst. Inc., Cary, NC). 
A final comparison was conducted using the Student t-test to assess if an adjusted guide resulted in a greater degree of error. The test showed that there was a difference at the base of the implant in the vestibular direction (Table 8). However, there was a somewhat similar deviation just in different directions. When no adjustments were required to seat the guide, there was a deviation toward the buccal (Figure 12). If the guide required adjustments, there was a similar error only in the lingual direction.

Table 8. Student's t-test assessing a difference in positioning if adjustment to the guide was required.

\begin{tabular}{|c|c|c|c|c|}
\hline \multicolumn{2}{|c|}{ Direction } & Difference & t-ratio & p-value \\
\hline \multirow{4}{*}{ 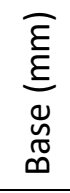 } & 3D offset & 0.15 & 0.72 & 0.4746 \\
\hline & Distal & 0.18 & 1.11 & 0.2723 \\
\hline & Vestibular & -0.64 & -2.49 & $0.0172 *$ \\
\hline & Apical & -0.13 & -0.33 & 0.7397 \\
\hline \multirow{4}{*}{$\begin{array}{l}\widehat{\underline{\xi}} \\
\underline{\underline{E}} \\
\stackrel{\varrho}{ }\end{array}$} & 3D offset & 0.33 & 1.38 & 0.1768 \\
\hline & Distal & 0.39 & 1.06 & 0.2944 \\
\hline & Vestibular & -0.52 & -1.41 & 0.1664 \\
\hline & Apical & -0.11 & -0.29 & 0.7698 \\
\hline \multicolumn{2}{|c|}{ Angle (degrees) } & 0.83 & 1.21 & 0.2330 \\
\hline
\end{tabular}

* p-values < 0.05 indicate significance (JMP/Pro Ver12, SAS Inst. Inc., Cary, NC).

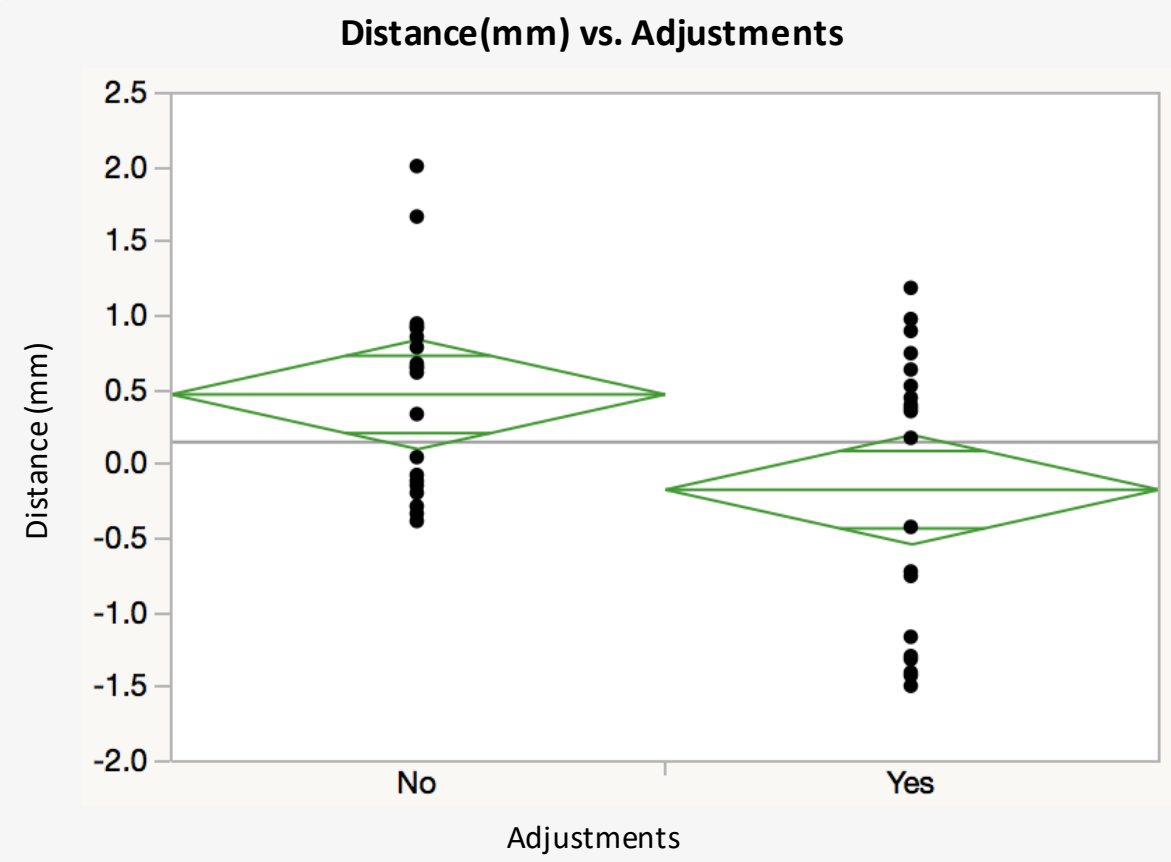

Figure 12. Difference in distance if guide required adjustments (JMP/Pro Ver12, SAS Inst. Inc., Cary, NC). 


\section{DISCUSSION}

When comparing the method of post-operative analysis, no significant difference in the data was found between using a CBCT or IOS scan bodies. This finding is valuable for future studies involving the clinical situation in patients. Where previously a post-operative $\mathrm{CBCT}$ would be required to assess accuracy of placement, an intraoral scan would suffice to relate the information for a comparison to the planned position. This would eliminate the need to radiographically expose the patient post-operatively for this area of research.

The findings of this study are comparable to the current literature regarding accuracy of implant placement using surgical guides (Table 9). The 3D offset was slightly larger in the current study at both the implant platform and apex. This could be attributed to the linear design of the surgical guide in the current study. The pig jaws were sectioned to be of suitable size to accommodate the FOV of the CBCT. This required that the animal model be divided into right and left quadrants. Rather than having a curved arch (like in a human jaw) to allow for tripodalized stabilization, the guides in this study were nearly linear to fit the porcine dentition. This could allow more tolerance of the guide to rock in a buccal/lingual direction.

In the current study, the planned positioning was developed to simulate an edentulous distal-extension, positioning the implants in an unbound space. This presents an additional challenge of maintaining the guide in a seated position. The guide could use the most distal tooth as a fulcrum while drilling the osteotomy through the guide, to result in discrepancies of the implant position. However, a true comparison in the mesial/distal and buccal/lingual direction is not possible, due to most of the literature assessing these measurements as purely lateral errors. The average apical error was more pronounced in the findings of the meta-analysis conducted by Zhou et al than in this study (Table 9) ${ }^{20}$. However, the angular error was slightly more in the current study. It is important to note that the meta-analysis included all three types of surgical guides (bone-, mucosa-, and tooth-supported), and included only prospective and retrospective studies. 
Table 9. Comparison of results of this study to a previous meta-analysis

\begin{tabular}{|c|c|c|c|c|}
\hline \multicolumn{3}{|c|}{ Direction } & Meta-Analysis ${ }^{20}$ & Current Study \\
\hline \multirow{4}{*}{$\begin{array}{l}\widehat{\xi} \\
\underline{\xi} \\
\stackrel{\nu}{n} \\
\tilde{D} \\
\infty\end{array}$} & \multicolumn{2}{|l|}{ 3D offset } & 1.25 & 1.43 \\
\hline & Distal & \multirow{2}{*}{ Lateral } & \multirow{2}{*}{1.05} & -0.18 \\
\hline & Vestibular & & & 0.15 \\
\hline & \multicolumn{2}{|l|}{ Apical } & 0.64 & -0.23 \\
\hline \multirow{4}{*}{$\begin{array}{l}\widehat{\underline{\varepsilon}} \\
\underline{\underline{E}} \\
\stackrel{\varrho}{\varrho}\end{array}$} & \multicolumn{2}{|l|}{ 3D offset } & 1.57 & 2.04 \\
\hline & Distal & \multirow{2}{*}{ Lateral } & \multirow{2}{*}{0.91} & -0.71 \\
\hline & Vestibular & & & 0.31 \\
\hline & \multicolumn{2}{|l|}{ Apical } & 1.24 & -0.17 \\
\hline \multicolumn{3}{|c|}{ Angle (degrees) } & 4.1 & 5.17 \\
\hline
\end{tabular}

It is of value to assess the combined intrinsic error indicated in the literature, when assessing the final discrepancies found in this study. When evaluating accuracy of actual measurements compared to digital measurements from the Carestream CS 9300, there was a slight over-estimation that averaged $0.229 \mathrm{~mm}^{25}$. Intraoral scanners have been found to have an average error of $0.0701 \mathrm{~mm}^{31}$. A study that evaluated accuracy of coDiagnostiX software, determined an average of $0.54 \mathrm{~mm}$ of error could be expected ${ }^{9}$. Furthermore, the FormLabs SLA printer can introduce another $0.100 \mathrm{~mm}$ of error ${ }^{2}$.

These cumulative errors can result in nearly a $1.0 \mathrm{~mm}$ error before even using the guide $(0.9391 \mathrm{~mm})$. This value does not factor in the tolerance that has been found between the guide sleeve and the drill key, or the drill key and the drill. The literature has shown that through calculations, a theoretical error can be determined depending on the implant length and tolerance measurement ${ }^{12}$. When assessing the Straumann surgical guide sleeve and drill components for placing a $14 \mathrm{~mm}$ implant, a theoretical $0.4 \mathrm{~mm}$ offset can be expected at the implant platform and a $1.0 \mathrm{~mm}$ offset can be expected at the implant apex ${ }^{12}$. This study used these calculations to find the percentage of error that was attributed to the intrinsic error of the sleeve. They found that sleeve tolerance was responsible for $62.6 \%$ of the error in implant placement ${ }^{12}$. 
When assessing the resulting positions of Implant A and Implant B, a significant difference was only found in implant depth. Implant A was consistently found to be slightly shorter than the planned depth, while Implant B was positioned slightly deeper than intended (Table 6, Figures 3 and 4). The reason for the discrepancy in this direction could be the material used for the surgical guide. It flexed slightly when pressure was applied while drilling at the more distal site (of Implant B) and could have contributed to the deeper placement and lower insertion torque values. Perhaps the flexure of the guide widened the osteotomy, resulting in reduced implant stability (as indicated by reduced insertion torque values). Assche et al also theorized that bending of the template could have occurred and affected the accuracy of implant positioning ${ }^{43}$. However, it is questionable whether an error in depth of placement is clinically significant. Since the goal of this study was to determine surgical guide accuracy, verification of depth of positioning was not evaluated during the procedures. During routine surgical procedures in a patient, depth of the osteotomy is usually assessed and compensated for as needed. Therefore, this difference may not be found under different surgical protocols.

The minimal difference in error between Implant A and Implant B is unusual, since the literature warns against placing implants too far from tooth support when utilizing a tooth-supported guide ${ }^{3 ; 43 ; 44}$. Naziri et al compared the accuracy of placing an implant in a tooth bound space to an unbounded space with a tooth-supported guide, and found there was significantly greater angular deviation in the unbounded space ${ }^{3}$. Other researchers used tooth/bone-supported guides to try to minimize error when placing an implant in an unbounded space ${ }^{43 ; 44}$. However, this approach requires the more invasive approach of laying a flap. Also, depending on the extent of the coverage with the bonesupport, additional errors can be incorporated by improper seating of the surgical guide.

These findings with regards to accuracy of positioning for Implant A compared to Implant B, carry valuable clinical significance. The designed guide did not rely on bone or mucosal coverage. Rather, it had a strut design connecting the drill sleeves. While a slight discrepancy in depth of positioning was observed, this finding can likely be compensated for in a clinical situation. Therefore, one can conclude that placing an implant further from tooth support does not compromise the positioning accuracy. 
When comparing accuracy between BL and BLT implants, significant differences were observed in the mesial/distal direction and in angular deviations (Table 7). BL implants were found to have a minimal distal error, while BLT implants had a larger distal error. However, the average distal deviation of BL implants was very minimal (Figure 5). Therefore, it can be concluded that BL implants had more accurate mesial/distal positioning accuracy. When assessing angulation, the BLT implants were significantly more accurate than the BL implants (Figure 6). Furthermore, another direction of measurement showed a tendency towards significance between the implant types $(\mathrm{p}$-value $=0.0643)$. The vestibular error showed a trend of lingual error for BL implants, and a buccal error for BLT implants (Figure 7). It is likely with a larger sample size, that this difference would show significance. The reason for these directional errors is unclear. However, the angulation difference could be explained by the drilling protocols of the two different systems and the structure of the different implant types. The tapered form of the BLT implants may have aligned more easily to the prepared osteotomy than the BL implants. Perhaps the use of a tap for the BL implants allowed a greater discrepancy when free-handing the implant into position. For a partially guided approach to implant placement (versus placing the implant through the surgical guide), the BLT implant may be the system of choice for improved angular accuracy.

The final variable assessed was surgical guide adjustment. When the guide needed to be adjusted to completely seat, there was a significantly greater deviation towards the lingual. However, when no adjustments were performed, there was a significant deviation towards the buccal (Table 8, Figure 8). The conclusion to be reached from these findings, is that adjusted guides were comparable in their inaccuracies to unadjusted guides. This information can provide re-assurance to the clinician who may worry that if the guide requires adjustment, the implant positioning will be less accurate.

Also, it was surprising that half of the guides required adjustment (Table 2). With the high accuracy of intraoral scanners and 3D printing, it can be expected that guides would seat without problems more consistently ${ }^{2 ; 31}$. However, the more intricate occlusal anatomy and shortened coronal height of the pig teeth may have contributed to the increased need for adjustments ${ }^{62}$. Pig molars in particular have an increased number of 
grooves, fossae, and eminences that were likely challenging for the Straumann CARES IOS to capture accurately ${ }^{62}$.

Further limitations of the study, include the limited sample size. Perhaps a larger sample size would have yielded additional significant differences of the variables assessed. Also, the researcher had limited experience utilizing a digital workflow, which could have led to marginally increased discrepancies.

Future areas of research can be focused on in vivo research, utilizing scan bodies to compare implant placement accuracy without the need for additional radiation. Furthermore, the Treatment Evaluation tool in coDiagnostiX provides a uniform method to assess implant placement accuracy. This can help provide more homogenous information, for more conclusive meta-analyses and systematic reviews. Also, the Straumann CARES IOS was capable of capturing the extended edentulous ridge with a powder technique. This may lead other researchers to push the boundaries of intraoral scanning in partially dentate and edentulous patients. 


\section{Chapter V: Summary and Conclusion}

\section{Summary}

This study selected pig jaws as an animal model to test the accuracy of implant placement with a tooth-supported guide. A digital workflow was used to collect diagnostic information to plan implant positioning and fabricate a surgical guide. The average error was more pronounced at both the base and tip of implants in the mesial direction. An average 3D offset of $1.43 \mathrm{~mm}$ was observed at the base, with a little higher average offset of $2.04 \mathrm{~mm}$ at the implant tip. Also, the average angular deviation was $5.17^{\circ}$.

\section{Conclusion}

1) Post-operative assessment using a CBCT or IOS scan bodies are comparable methods to evaluate planned versus placed implant positioning.

2) Flexure of the surgical guide can cause implants placed further across an edentulous span to be positioned deeper than planned.

3) BLT implants have better angular accuracy than BL implants with a partially guided surgical approach.

4) Adjustments, to fully seat a guide on the dentition, do not affect the accuracy of implant placement.

5) The accuracy of implant positioning utilizing a digital workflow is consistent with findings in the literature. 


\section{References}

1. Elian N. 2009. Dental implants: Past, present, and future. Compendium. p. 456-458.

2. Whitley D, Bencharit S. 2015. Digital implantology with desktop 3d printing.: Formlabs White Paper. p. 1-15.

3. Naziri E, Schramm A, Wilde F. 2016. Accuracy of computer-assisted implant placement with insertion templates. GMS Interdiscip Plast Reconstr Surg DGPW. 5:Doc15.

4. Scherer MD. 2014. Presurgical implant-site assessment and restoratively driven digital planning. Dent Clin North Am. 58(3):561-595.

5. Dreiseidler T, Tandon D, Kreppel M, Neugebauer J, Mischkowski RA, Zinser MJ, Zöller JE. 2012. Cbct device dependency on the transfer accuracy from computeraided implantology procedures. Clin Oral Implants Res. 23(9):1089-1097.

6. Mora MA, Chenin DL, Arce RM. 2014. Software tools and surgical guides in dentalimplant-guided surgery. Dent Clin North Am. 58(3):597-626.

7. Gallardo YR, Bohner L, Tortamano P, Pigozzo MN, Laganá DC, Sesma N. 2018. Patient outcomes and procedure working time for digital versus conventional impressions: A systematic review. J Prosthet Dent. 119(2):214-219.

8. Mangano F, Gandolfi A, Luongo G, Logozzo S. 2017. Intraoral scanners in dentistry: A review of the current literature. BMC Oral Health. 17(1):149.

9. Flügge T, Derksen W, Te Poel J, Hassan B, Nelson K, Wismeijer D. 2017.

Registration of cone beam computed tomography data and intraoral surface scans - a prerequisite for guided implant surgery with cad/cam drilling guides. Clin Oral Implants Res. 28(9):1113-1118.

10. Schneider D, Schober F, Grohmann P, Hammerle CH, Jung RE. 2015. In-vitro evaluation of the tolerance of surgical instruments in templates for computer-assisted guided implantology produced by 3-d printing. Clin Oral Implants Res. 26(3):320325.

11. Farley NE, Kennedy K, McGlumphy EA, Clelland NL. 2013. Split-mouth comparison of the accuracy of computer-generated and conventional surgical guides. Int J Oral Maxillofac Implants. 28(2):563-572.

12. Cassetta M, Di Mambro A, Giansanti M, Stefanelli LV, Cavallini C. 2013. The intrinsic error of a stereolithographic surgical template in implant guided surgery. Int J Oral Maxillofac Surg. 42(2):264-275. 
13. Kühl S, Zürcher S, Mahid T, Müller-Gerbl M, Filippi A, Cattin P. 2013. Accuracy of full guided vs. Half-guided implant surgery. Clin Oral Implants Res. 24(7):763-769.

14. Jacobs R, Quirynen M. 2014. Dental cone beam computed tomography: Justification for use in planning oral implant placement. Periodontol 2000. 66(1):203-213.

15. Hatcher DC, Dial C, Mayorga C. 2003. Cone beam ct for pre-surgical assessment of implant sites. J Calif Dent Assoc. 31(11):825-833.

16. Wyatt CC, Pharoah MJ. 1998. Imaging techniques and image interpretation for dental implant treatment. Int J Prosthodont. 11(5):442-452.

17. Hassan B, Couto Souza P, Jacobs R, de Azambuja Berti S, van der Stelt P. 2010. Influence of scanning and reconstruction parameters on quality of three-dimensional surface models of the dental arches from cone beam computed tomography. Clin Oral Investig. 14(3):303-310.

18. Koenig LJ. 2015. Imaging of the jaws. Semin Ultrasound CT MR. 36(5):407-414.

19. Scarfe WC, Farman AG. 2008. What is cone-beam ct and how does it work? Dent Clin North Am. 52(4):707-730, v.

20. Zhou W, Liu Z, Song L, Kuo CL, Shafer DM. 2018. Clinical factors affecting the accuracy of guided implant surgery-a systematic review and meta-analysis. J Evid Based Dent Pract. 18(1):28-40.

21. Araki K, Patil S, Endo A, Okano T. 2013. Dose indices in dental cone beam ct and correlation with dose-area product. Dentomaxillofac Radiol. 42(5):20120362.

22. Affairs ADACoS. 2012. The use of cone-beam computed tomography in dentistry: An advisory statement from the american dental association council on scientific affairs. J Am Dent Assoc. 143(8):899-902.

23. Hatcher DC. 2010. Operational principles for cone-beam computed tomography. J Am Dent Assoc. 141 Suppl 3:3S-6S.

24. Widmann G, Fischer B, Berggren JP, Dennhardt A, Schullian P, Reto B, Puelacher W. 2016. Cone beam computed tomography vs multislice computed tomography in computer-aided design/computer-assisted manufacture guided implant surgery based on three-dimensional optical scanning and stereolithographic guides: Does image modality matter? Int J Oral Maxillofac Implants. 31(3):527-533.

25. Accuracy and reproducibility of four cone beam computed tomography devices using 3d implant-planning software. 
26. Visconti MA, Verner FS, Assis NM, Devito KL. 2013. Influence of maxillomandibular positioning in cone beam computed tomography for implant planning. Int J Oral Maxillofac Surg. 42(7):880-886.

27. Richert R, Goujat A, Venet L, Viguie G, Viennot S, Robinson P, Farges JC, Fages M, Ducret M. 2017. Intraoral scanner technologies: A review to make a successful impression. J Healthc Eng. 2017:8427595.

28. Punj A, Bompolaki D, Garaicoa J. 2017. Dental impression materials and techniques. Dent Clin North Am. 61(4):779-796.

29. Ender A, Attin T, Mehl A. 2016. In vivo precision of conventional and digital methods of obtaining complete-arch dental impressions. J Prosthet Dent. 115(3):313320.

30. Nedelcu R, Olsson P, Nyström I, Rydén J, Thor A. 2018. Accuracy and precision of 3 intraoral scanners and accuracy of conventional impressions: A novel in vivo analysis method. J Dent. 69:110-118.

31. Park JM. 2016. Comparative analysis on reproducibility among 5 intraoral scanners: Sectional analysis according to restoration type and preparation outline form. J Adv Prosthodont. 8(5):354-362.

32. Rosenfeld AL, Mandelaris GA, Tardieu PB. 2006. Prosthetically directed implant placement using computer software to ensure precise placement and predictable prosthetic outcomes. Part 3: Stereolithographic drilling guides that do not require bone exposure and the immediate delivery of teeth. Int J Periodontics Restorative Dent. 26(5):493-499.

33. Whitley D, Eidson RS, Rudek I, Bencharit S. 2017. In-office fabrication of dental implant surgical guides using desktop stereolithographic printing and implant treatment planning software: A clinical report. J Prosthet Dent. 118(3):256-263.

34. Tahayeri A, Morgan M, Fugolin AP, Bompolaki D, Athirasala A, Pfeifer CS, Ferracane JL, Bertassoni LE. 2018. 3d printed versus conventionally cured provisional crown and bridge dental materials. Dent Mater. 34(2):192-200.

35. Reyes A, Turkyilmaz I, Prihoda TJ. 2015. Accuracy of surgical guides made from conventional and a combination of digital scanning and rapid prototyping techniques. J Prosthet Dent. 113(4):295-303.

36. Neumeister A, Schulz L, Glodecki C. 2017. Investigations on the accuracy of 3dprinted drill guides for dental implantology. Int J Comput Dent. 20(1):35-51. 
37. Deeb GR, Allen RK, Hall VP, Whitley D, Laskin DM, Bencharit S. 2017. How accurate are implant surgical guides produced with desktop stereolithographic 3dimentional printers? J Oral Maxillofac Surg. 75(12):2559.e2551-2559.e2558.

38. Noharet R, Pettersson A, Bourgeois D. 2014. Accuracy of implant placement in the posterior maxilla as related to 2 types of surgical guides: A pilot study in the human cadaver. J Prosthet Dent. 112(3):526-532.

39. Park C, Raigrodski AJ, Rosen J, Spiekerman C, London RM. 2009. Accuracy of implant placement using precision surgical guides with varying occlusogingival heights: An in vitro study. J Prosthet Dent. 101(6):372-381.

40. Park SJ, Leesungbok R, Cui T, Lee SW, Ahn SJ. 2017. Reliability of a cad/cam surgical guide for implant placement: An in vitro comparison of surgeons' experience levels and implant sites. Int J Prosthodont. 30(4):367-169.

41. Lee DH, An SY, Hong MH, Jeon KB, Lee KB. 2016. Accuracy of a direct drillguiding system with minimal tolerance of surgical instruments used for implant surgery: A prospective clinical study. J Adv Prosthodont. 8(3):207-213.

42. Choi M, Romberg E, Driscoll CF. 2004. Effects of varied dimensions of surgical guides on implant angulations. J Prosthet Dent. 92(5):463-469.

43. Van Assche N, van Steenberghe D, Guerrero ME, Hirsch E, Schutyser F, Quirynen M, Jacobs R. 2007. Accuracy of implant placement based on pre-surgical planning of three-dimensional cone-beam images: A pilot study. J Clin Periodontol. 34(9):816-821.

44. Dreiseidler T, Neugebauer J, Lingohr T, Rothamel D, Mischkowski RA, Zöller JE. 2009. Accuracy of a newly developed integrated system for dental implant planning. Clinical oral implants research. p. 1191 - 1199.

45. Cassetta M, Stefanelli LV, Giansanti M, Calasso S. 2012. Accuracy of implant placement with a stereolithographic surgical template. Int J Oral Maxillofac Implants. 27(3):655-663.

46. Cassetta M, Giansanti M, Di Mambro A, Calasso S, Barbato E. 2013. Accuracy of two stereolithographic surgical templates: A retrospective study. Clin Implant Dent Relat Res. 15(3):448-459.

47. Turbush SK, Turkyilmaz I. 2012. Accuracy of three different types of stereolithographic surgical guide in implant placement: An in vitro study. J Prosthet Dent. 108(3):181-188. 
48. Ozan O, Turkyilmaz I, Ersoy AE, McGlumphy EA, Rosenstiel SF. 2009. Clinical accuracy of 3 different types of computed tomography-derived stereolithographic surgical guides in implant placement. J Oral Maxillofac Surg. 67(2):394-401.

49. Bover-Ramos F, Viña-Almunia J, Cervera-Ballester J, Peñarrocha-Diago M, GarcíaMira B. 2018. Accuracy of implant placement with computer-guided surgery: A systematic review and meta-analysis comparing cadaver, clinical, and in vitro studies. Int J Oral Maxillofac Implants. 33(1):101-115.

50. Otto S, Pautke C, Martin Jurado O, Nehrbass D, Stoddart MJ, Ehrenfeld M, Zeiter S. 2017. Further development of the mronj minipig large animal model. J Craniomaxillofac Surg. 45(9):1503-1514.

51. Aerssens J, Boonen S, Lowet G, Dequeker J. 1998. Interspecies differences in bone composition, density, and quality: Potential implications for in vivo bone research. Endocrinology. 139(2):663-670.

52. Erdogan Ö, Üstün Y, Tatli U, Damlar I, Daglıglu K. 2013. A pig model for the histomorphometric evaluation of hard tissue around dental implants. J Oral Implantol. 39(5):551-557.

53. Litten-Brown JC, Corson AM, Clarke L. 2010. Porcine models for the metabolic syndrome, digestive and bone disorders: A general overview. Animal. 4(6):899-920.

54. Ruehe B, Niehues S, Heberer S, Nelson K. 2009. Miniature pigs as an animal model for implant research: Bone regeneration in critical-size defects. Oral Surg Oral Med Oral Pathol Oral Radiol Endod. 108(5):699-706.

55. Ohtani T, Kusumoto N, Wakabayashi K, Yamada S, Nakamura T, Kumazawa Y, Yatani H, Sohmura T. 2009. Application of haptic device to implant dentistry-accuracy verification of drilling into a pig bone. Dent Mater J. 28(1):75-81.

56. Pautke C, Kreutzer K, Weitz J, Knödler M, Münzel D, Wexel G, Otto S, Hapfelmeier A, Stürzenbaum S, Tischer T. 2012. Bisphosphonate related osteonecrosis of the jaw: A minipig large animal model. Bone. 51(3):592-599.

57. Mosekilde L, Weisbrode SE, Safron JA, Stills HF, Jankowsky ML, Ebert DC, Danielsen CC, Sogaard CH, Franks AF, Stevens ML. 1993. Calcium-restricted ovariectomized sinclair s-1 minipigs: An animal model of osteopenia and trabecular plate perforation. Bone. 14(3):379-382.

58. Stadlinger B, Hennig M, Eckelt U, Kuhlisch E, Mai R. 2010. Comparison of zirconia and titanium implants after a short healing period. A pilot study in minipigs. Int $\mathbf{J}$ Oral Maxillofac Surg. 39(6):585-592. 
59. Rocchietta I, Schupbach P, Ghezzi C, Maschera E, Simion M. 2012. Soft tissue integration of a porcine collagen membrane: An experimental study in pigs. Int $\mathbf{J}$ Periodontics Restorative Dent. 32(1):e34-40.

60. Štembírek J, Kyllar M, Putnová I, Stehlík L, Buchtová M. 2012. The pig as an experimental model for clinical craniofacial research. Lab Anim. 46(4):269-279.

61. Carmagnola D, Abati S, Addis A, Ferrieri G, Chiapasco M, Romeo E, Vogel G. 2009. Time sequence of bone healing around two implant systems in minipigs: Preliminary histologic results. Int J Periodontics Restorative Dent. 29(5):549-555.

62. Wang S, Liu Y, Fang D, Shi S. 2007. The miniature pig: A useful large animal model for dental and orofacial research. Oral Dis. 13(6):530-537. 


\title{
Curriculum Vitae
}

\author{
Nicole Andreini
}

\section{Objective:}

To provide high quality, comprehensive care to patients by performing implant, removable, and fixed prosthodontics.

\section{Education:}

West Virginia Graduate Prosthodontics - Morgantown, WV

Master of Science in Prosthodontics

2015 - present

West Virginia University School of Dentistry - Morgantown, WV

Doctor of Dental Surgery

$2011-2015$

West Liberty University - West Liberty, WV

Non-Degree Seeking

$2010-2011$

West Virginia Wesleyan College - Buckhannon, WV

Bachelor of Science in Biology, Minor in Chemistry

$2008-2010$

Grove City College - Grove City, PA

Major in Molecular Biology

$2006-2007$

\section{Professional Experience:}

Resident Teaching at WVU. Morgantown, WV

Predoctoral Student Clinics

$8 / 17$ - present

Supervise dental students during their clinical procedures.

Introduction to Implant Dentistry DENT 754

Lecturer and Lab Director

Lab Instructor

$8 / 17$ - present

Occlusion DENT 731

Lecturer and Lab Instructor

$5 / 17-8 / 17$

Drs. Perrine Dental Practice. Cowen, WV

Rural Rotation

$3 / 15-5 / 15$

Gained dental experience treating patients in a private practice office.

Dr. Thomas Leslie and Associates Dental Practice. Berkeley Springs, WV

Rural Rotation

Provided dental care to patients under supervising dentists. Learned additional dental

techniques and office management practices.

West Liberty Dental Internship. Wheeling, WV

Dental Intern

$8 / 10-5 / 11$

Observed and assisted in the dental offices of local practitioners.

Dr. Johns and Conde Dental Practice. Buckhannon, WV

Dental Intern

$1 / 10-5 / 10$

Spent time assisting student dentists on their rural rotation. Learned about dental

materials, sterilization techniques, and managing a practice.

Dr. Andreini Urological Practice. Wheeling, WV

Assistant Business Manager

Filed documents and performed other clerical work.

$5 / 03-5 / 06$ 


\section{Licensure:}

West Virginia Board of Dentistry

2015 - present SRTA/ADEX clinical board exam

\section{Research:}

Predoctoral - Probiotic Internet Database - Bac2Health.from-ca.com

Researched the various applications of probiotic supplements and the strength of evidence to support the use of different strains.

Undergraduate - Senior Research Paper 2010

Reviewed the research on tissue engineering for periodontitis using modified mesenchymal stem cells.

\section{Certifications:}

Basic Cardiac Life Support - Renewal 2017

Academy of Laser Dentistry - Diode, Er:YAG 2015

\section{Clinical Presentations:}

Andreini NI and Bryington MS. Management of Peri-implant Induced Jaw Fracture by Single Implant Retained Overdenture. Poster Presentation: ACP Annual Session - San Diego, CA 2016.

\section{CE Attended:}

Creativity with New Ceramics

Restorative Dentistry and Material Options

Posterior Composites: A Rapid, Simplified, and Predictable Placement Technique and Metal-Free Crowns

Emergency Medicine in Dentistry

Advanced Complete Dentures

Mixed Media Dental Solutions Using IPS emax

Drug Diversion and Best Practice Prescribing Controlled Substances

Esthetics and Function in Implant Dentistry: Treatment Planning, Surgical and

Restorative Aspects

\section{Professional Meetings:}

American College of Prosthodontics - San Francisco, CA 2017

Straumann Resident Symposium - Andover, MA 2017

Academy of Osseointegration -Orlando, FL 2017

Digital Dentistry Symposium - Chicago, IL 2017

American Academy of Fixed Prosthodontics - Chicago, IL 2017

Chicago Dental Society Midwinter Meeting - Chicago, IL 2017

LMT Lab Day - Chicago, IL $\quad 2017$

American College of Prosthodontics - San Diego, CA 2016

WV Dental Association Annual Meeting - White Sulfur Springs, WV 2016

ITI Symposium - Chicago, IL $\quad 2016$

Academy of Osseointegration -San Diego, CA 2016

Straumann Symposium - Boston Massachussetts 2016

American Academy of Fixed Prosthodontics - Chicago, IL 2016

LMT Lab Day - Chicago, IL 2016 
American College of Prosthodontics - Orlando, FL

Memberships:

WVU Graduate Prosthodontics

WVU Prosthodontic Club

2017 - present

ACP Member

2015 - present

ITI Member

2015 - present

WVU School of Dentistry

Student Research Group

$2011-2015$

Student Advisory Board

$2013-2015$

American Student Dental Association

$2011-2015$

Pediatric Dental Club

$2011-2015$

\section{Honors and Awards:}

WVU Graduate Prosthodontics

Chief Resident

Rising Star Award

WVU School of Dentistry

Dental Implant Student Award

American Association of OMS

Outstanding Achievement and Excellence in Prosthodontics

Southeastern Academy of Prosthodontics

Golden Key International Honour Society

Honors Society

WV Wesleyan College

Omicron Delta Kappa National Leadership Honor Society 2010

Who's Who Among Students

Beta Beta Beta Biological Honor Society

Grove City College

Crown and Sceptre Honor Society

\section{Community Involvement:}

Dental Awareness Projects

Participated in organized service to promote dental education in the community.

- "Destination Dentistry"

- "Brush with Dentistry"

- Horseshoe Adventure Camp for low-income children

- "Baby and Me Day" at Meadowbrook Mall

- Toys and Tots Day Care

- Health Fair at Unity Manor

- Governor's Honors Academy seminar at WVU School of Dentistry

- Miles for Smiles 5K Run

Project MUSHROOM (Multidisciplinary Unsheltered Homeless Relief Outreach of Morgantown) Provided medical care and food to the homeless in Morgantown, WV.

Vietnam Mission Trip Fundraising 
Raised money used to buy dental supplies and pay for dental care for children in Vietnam.

\section{Relay for Life}

Participated in a fundraising team to contribute to cancer research by getting sponsors and collecting donations. 